\title{
Un apprentissage de la révolution : Marx et la conjoncture 1848-1851
}

\section{Antoine Janvier}

\section{OpenEdition}

\section{Journals}

Édition électronique

URL : http://journals.openedition.org/grm/121

DOI : 10.4000/grm. 121

ISSN : 1775-3902

\section{Éditeur}

Groupe de Recherches Matérialistes

\section{Référence électronique}

Antoine Janvier, « Un apprentissage de la révolution : Marx et la conjoncture 1848-1851 », Cahiers du GRM [En ligne], 1 | 2011, mis en ligne le 28 mars 2011, consulté le 01 mai 2019. URL : http:// journals.openedition.org/grm/121; DOI : 10.4000/grm.121 


\section{UN APPRE NTISSAGE DE LA RÉVOLUTION : MARX ET L A CONJONCTURE 1848- 1851}

ANTOINE JANVIER

\section{Introduction : 1848 de la farce au tragique}

Dans le tableau marxiste des insurrections révolutionnaires françaises, 1848 fait figure d'événement superficiel $^{1}$ : cette fois, contrairement à la grande Révolution de 1789 et à l'insurrection de 1870, pas de Commune. Face à ce manque de profondeur de l'événement, Marx semble railler les gesticulations dérisoires de ses agents, par contraste avec la grandeur des acteurs de 1789. Les premières phrases du Dix-huit Brumaire de Louis Bonaparte sont restées célèbres :

« Hegel fait quelque part cette remarque que tous les grands événements et personnages historiques se répètent pour ainsi dire deux fois. Il a oublié d'ajouter : la première fois comme tragédie, la seconde fois comme farce. Caussidière pour Danton, Louis Blanc pour Robespierre, la Montagne de 1848 à 1851 pour la Montagne de 1793 à 1795, le neveu pour l'oncle $»^{2}$

Ce texte est issu d'un exposé présenté avec Stéphane Legrand, à qui il doit beaucoup.

2 K. Marx, Le Dix-huit Brumaire de Louis Bonaparte, tr. fr. Marcel Ollivier, éd.

E. Barot et J.-N. Ducange, Paris, Livre de Poche, 2007, p. 117-118. 
Mais la conjoncture février-juin 1848 et, plus largement, 18481851, fut aussi, à sa manière, une conjoncture tragique. Car elle connut avant tout la victoire de la contre-révolution : de l'écrasement du prolétariat durant les journées de juin au coup d'État du président Bonaparte et à la renaissance de l'Empire sous la férule autoritaire de Napoléon III, les trois années de troubles politiques sont marquées du sceau de la réaction. C'est le moment du pour-soi du prolétariat, désormais isolé et opposé à la bourgeoisie au prix d'une désillusion cruelle. À bien des égards, l'histoire de 1848 est l'histoire de la classe ouvrière aveugle, menée tel un troupeau vers l'abattoir par une bourgeoisie cynique et corrompue. Mystification ouvrière qui répète, en coulisse, 1789 sur le mode tragique, et à laquelle répond une mystification petite-bourgeoise naïve et comique, qui répète quant à elle, sur le devant de la scène, 1789 sur le mode de la farce. La répétition entre 1789 et 1848 se dédouble donc à l'intérieur même de 1848, pour en dessiner la profondeur. À ce titre, les événements ne peuvent plus être réduits à leur seule dimension idéologique et, encore moins, aux illusions qu'ils ont générées. La représentation théâtrale eut lieu pour quelqu'un, et les acteurs avaient beau jouer, il leur arrivait de descendre de la scène et d'en sentir l'effet. Une lecture attentive des textes marxiens sur la conjoncture nous convainc de corriger l'image d'Épinal d'une période de l'histoire réduite à une comédie grotesque. Avant l'analyse socio-économique du Dix-huit Brumaire, paru en 1852, Marx procède à un travail d'investigation journalistique et historique, presque à chaud, en tout cas à même les événements. Il éprouve alors pour son propre compte la dimension tragique de la conjoncture. Les quatre articles qu'il en retire - dont il publiera les trois premiers dans la Nouvelle gazette rhénane, de janvier à mars 1850 - seront rassemblés par Engels en 1895 sous le titre Les luttes de classes en France. L'ouverture du premier article ne laisse aucun doute sur le sens accordé par Marx à la révolution de 1848 :

«À l'exception de quelques rares chapitres, chaque période importante des annales de la révolution de 1848 à 1849 a pour titre: Défaite de la révolution! Ce qui succomba dans ces 
défaites, ce n'est pas la révolution. Ce sont les traditionnels accessoires pré-révolutionnaires [...]. En un mot : ce n'est pas par ses conquêtes tragicomiques immédiates que la révolution a progressé et s'est frayée la voie ; bien au contraire, en produisant une contre-révolution massive, puissante, en suscitant un adversaire à combattre, le parti de la subversion s'est mué en un parti réellement révolutionnaire ». ${ }^{1}$

Ainsi s'agit-il aussi d'une tragédie, plus exactement d'une " tragicomédie », dont Marx rend compte tout en s'efforçant de dégager les effets de réalité des illusions des uns et des autres, et de convertir le sentiment de la défaite en une expérience de lutte, porteuse de victoires à venir. Dans cette mesure, il est conduit à un double geste paradoxal : d'un côté, il s'agit de ressaisir le sens réel des événements derrière leur superficialité, autrement dit de les relire à la lumière du cours de l'histoire et de la dialectique que le matérialisme peut dégager ; de l'autre, il s'agit, précisément pour éprouver l'épaisseur réelle de l'ensemble de la conjoncture, de descendre dans le chaudron de l'histoire en train de se faire, de prendre la mesure de son indétermination et de sa contingence, et de rejouer les actes - aux deux sens du terme - dans leur effectivité. C'est alors que la nécessité dialectique se relâche et que les événements, pour reprendre une formule utilisée à plusieurs reprises par Marx lui-même, semblent se précipiter (beschleunigen) ${ }^{2}$.

Les luttes de classes en France ne déploie l'armature théorique de la dialectique historique - exposée dans le Manifeste du parti communiste paru quelques jours avant les événements ${ }^{3}-$ qu'en la plongeant dans la masse multiple et contingente des faits recensés.

1 K. Marx, Les Luttes de classes en France, trad. Maximilien Rubel, Paris, Gallimard, Folio histoire, 2002, p. 9.

Voir par exemple ibid., p. 15. Le terme revient à plusieurs reprises sous la plume de Marx.

3 Marx devait en effet livrer le texte au comité central de la Ligue des communistes pour le 1er février 1848. L'insurrection parisienne démarra le 22 février. Voir la notice au Manifeste dans Karl Marx, Euvres I, Économie I, éd. M. Rubel, Paris, Gallimard, La Pléiade, 1963, p. 159. 
Pour épouser la complexité de l'histoire, Marx complique le modèle dialectique. Les luttes de classes en France s'offre à nous comme le lieu d'un nœud improbable entre deux tendances incompatibles : d'une part une lecture dialectique de la conjoncture, qui en fait un moment nécessaire du cours de l'histoire des luttes de classes ; d'autre part une lecture non-dialectique de la conjoncture, en termes de conjonctions contingentes de forces dont l'action peut toujours précipiter le devenir, sinon révolutionnaire, du moins insurrectionnel ${ }^{1}$. L'objectif que se propose le texte qui va suivre est de relever les différents motifs de cette dualité interprétative, pour en identifier les enjeux théoriques et pratiques. À cette fin, nous nous arrêterons sur quelques temps forts de l'analyse marxienne, en essayant d'autre part de les articuler à certains motifs issus de la constellation 《blanquiste $»^{2}$, qui projette une théorie divergente de l'action, pensée à partir de son urgence, de son indétermination et de sa contingence.

\section{Des causes aux événements}

Il n'est pas inutile de rappeler que, contrairement au Dix-huit Brumaire, quand Marx écrit les articles repris dans Les luttes de classes en France, Louis-Napoléon Bonaparte n'est pas encore Napoléon III. Autrement dit, la conjoncture n'est pas close. Or Marx replace précisément l'indétermination de l'issue de la situation au cœur de sa compréhension des années précédentes, dès le début des faits et au moment même où il écrit. Car sans la prise en compte de cette dimension inchoative de la conjoncture, l'explosion insurrectionnelle de février 1848 demeure incompréhensible. Les

1 On peut y voir l'effet d'une dualité de statut du texte - des textes - de Marx : entre analyse théorique et écrit de conjoncture.

2 Selon la suggestion d'Alix Héricord, dans sa présentation du livre de F. Pardigon, Episodes des journées de juin 1848, Paris, La Fabrique, 2008, intitulée «Une mémoire d'outre-tombe », voir en particulier p. 28-33. 
causes seules ne suffisent pas à l'expliquer, pas plus qu'elles n'expliquent la suite des événements de la conjoncture 1848-1851.

Dès les premières pages des Luttes de classes en France, Marx dénombre les causes de la révolution de Février, conçues comme autant de contradictions motrices. Tout porte d'abord à croire que c'est leur accumulation et leur totalisation dialectique qui provoqua leur radicalisation, et du même coup l'explosion qui s'ensuivit : contradictions économique et politique interne à la classe bourgeoise entre aristocratie financière au pouvoir depuis juillet 1830 et bourgeoisie foncière et industrielle dans l'opposition; contradiction entre un pouvoir au faste de plus en plus dispendieux et une population dans un état de dénuement de plus en plus criant $^{1}$; contradiction entre le mode de vie de l'aristocratie financière et « les lois bourgeoises elles-mêmes $»^{2}$; contradiction entre les intérêts économiques de cette même aristocratie financière et les conditions par lesquelles ces intérêts peuvent être satisfaits, à savoir l'État (ce qui détermine une contradiction secondaire entre l'endettement de l'État et l'équilibre budgétaire permettant non seulement son maintien, mais également cet endettement même) ; contradiction entre une politique étrangère d'alliance avec les puissances réactionnaires et un sentiment populaire nationaliste mais favorable aux soulèvements en Europe.

Cependant, malgré l'importance et les effets réels de ces contradictions, Marx ne s'en satisfait pas : si les causes sont nécessaires, elles ne sont pas suffisantes. Continuons à prendre pour exemple privilégié l'analyse du bouleversement de Février. On peut bien dénombrer à l'infini toutes ces contradictions, leur accumulation ne permet pas pour autant de rendre raison des soulèvements qui, les 22, 23 et 24 février 1848, semblent résulter de leur conjonction. Car,

Voir K. Marx, Les Luttes de classes en France, op. cit., p. 15 : «D'un côté, les orgies éhontées de l'aristocratie financière, de l'autre, la lutte du peuple pour les denrées de première nécessité. À Buzançais, on exécuta les émeutiers de la faim, à Paris des escrocs repus furent soustraits aux tribunaux par la famille royale. »

Ibid., p. 13. 
à ne s'en tenir qu'au relevé des causes recensées, on ne rend pas pour autant raison des motifs déterminant la subite entente des différents opposants à la Monarchie de Juillet, pas plus que du moment spécifique de l'explosion insurrectionnelle. Concernant le premier point, si toutes les classes sont opposées au pouvoir, elles ne le sont pas pour les mêmes raisons, à tel point que les types d'expression de cette opposition varient $\mathrm{du}$ tout au tout. En l'état, il est incompréhensible que les motifs d'opposition convergent pour créer une unité insurrectionnelle. D'autre part, on peut tout de même se demander pourquoi, après tout, la révolution de Février surgit à ce moment et pas deux ou trois ans auparavant, ou même quelques mois, puisque la plupart des causes recensées agissaient déjà. Fin février 1848 , c'est à la fois fort tôt du point des motifs et fort tard du point de vue des conditions. Fort tôt pour les raisons susdites : il est loin d'être évident que les motifs de la bourgeoisie industrielle, la petite-bourgeoisie et le prolétariat convergent, c'est-à-dire que les conditions d'un rassemblement effectif et conséquent soient remplies. Fort tard, parce que les frasques de l'aristocratie financière, son mode de fonctionnement, sa politique étrangère et le conflit qui l'oppose à la bourgeoisie industrielle ne datent pas de février 1848, loin de là.

Ce que nous identifions ici avec Marx, c'est l'insuffisance d'une lecture mécaniciste de l'insurrection, comme si l'on résistait ou se soulevait en raison d'intérêts objectifs, comme si la révolte d'un peuple était la résultante pure et simple de contradictions. Les mouvements insurrectionnels ou les mouvements de résistance ne sont jamais l'effet mécanique des rapports sociaux existants et des intérêts objectifs que ces rapports déterminent. En d'autres termes : les raisons qui font qu'on se révolte ne sont jamais les raisons qu'on aurait de se révolter. On ne se révolte jamais pour les bonnes raisons, celles qui sont théoriquement explicables en termes d'intérêt objectif. Pourquoi ? C'est que l'ordre social et les rapports sociaux réglés contre lesquels on se révolte, ne peuvent être tels, c'est-à-dire ordonnés et réglés, ne peuvent exercer une domination régulière et durable que parce que leurs sont consubstantiels certains mécanismes 
par lesquels ils obtiennent l'adhésion de ceux-là même qui auraient intérêt à les transformer ou à les renverser. C'est ce qui résulte du concept même de luttes des classes comme constitutives de la formation sociale. La lutte est donnée comme une coordonnée de la formation sociale. Il en découle que la loi de l'ordre social, en tant qu'ordination de la lutte des classes, c'est d'entraîner mécaniquement la tendance des classes dominées à ne pas lutter en vue de leurs intérêts. En résumé, les conditions de l'adhésion des dominés au système qui perpétue leur domination sont toujours déjà données dans une structure sociale, ou plus précisément : les stratégies de dépolitisation qui effacent la conflictualité du social, aussi bien que les mouvements de politisation qui s'efforcent de l'y introduire sont des moments de la lutte.

On en trouvera une confirmation frappante dans les propos de l'insurgé de juin François Pardigon en préface à ses Épisodes des journées de juin $1848^{1}$ : à la limite, pour un opposant à un système donné, le plus dur n'est pas, au sein d'une structure sociale, la lutte dans la rue pour la république et contre les formes d'oppression bourgeoise ; le plus dur, la lutte « la plus rude », c'est celle qui va à l'encontre des conditions sociales d'existence qui attachent tout homme à un système déterminé d'intérêts.

«Qu'on se représente les mille obstacles qui font barrière à l'émancipation de l'homme jeune au moment où il sort de la vie de famille pour entrer dans la vie sociale. De tous les combats, c'est le plus meurtrier pour la démocratie, car c'est celui qui lui enlève le plus d'adeptes. L'autorité paternelle, l'affection de la mère, l'entourage des proches et des amis, la somme totale des intérêts, concluent à la routine, et opposent, sans vergogne, à la morale peu lucrative des intérêts et des

Pardigon était étudiant au moment de l'insurrection de1848. Il rejoint les insurgés lors des journées de juin. Arrêté durant la majeure partie des faits, il les raconte du point de vue de celui qui n'en voit rien, ou très peu, mais qui devine et subit. Il sera par la suite déporté puis émigré à Londres et aux ÉtatsUnis. Nous revenons plus loin, dans l'appendice, sur quelques motifs de son récit, d'une force théorique et poétique incontestable. 
droits généraux la morale plus stimulante des intérêts particuliers, des immunités personnelles ». ${ }^{1}$

Morale qui est d'emblée donnée avec la structure sociale comme ce qui conditionne toujours déjà en son sein l'adhésion des dominés à l'ordre qu'elle reproduit - et donc à la place qu'ils sont déterminés à venir y occuper et dans laquelle ils sont conduits, pour exister comme sujets, à se reconnaître. Par conséquent, les conditions d'un refus du système relèvent par définition de ce qui ne peut pas être donné à l'intérieur du système en tant que tel. Dit autrement : les causes qu'on peut toujours recenser ne suffisent pas à rendre raison d'une explosion insurrectionnelle, d'un événement révolutionnaire.

La faible valeur épistémologique des causes de Février tiendraitelle à leur relative superficialité par rapport à ce qui détermine réellement, et en dernière instance, le cours de l'histoire aux yeux de Marx : la situation économique mondiale ? En effet, Marx repère deux événements qui semblent être, « finalement », les véritables raisons de 1848. Ce sont " deux événements économiques de portée mondiale $\gg^{2}$ : premièrement la disette issue de problèmes agricoles ; deuxièmement la crise générale du commerce et de l'industrie en Angleterre, issue des revers subis par les spéculateurs en actions des chemins de fer. Cependant, il ne faut pas pour autant comprendre que ces événements seraient des causes plus «profondes » que celles évoquées plus haut. Justement parce que ce ne sont pas, à proprement parler, des causes. De ces deux événements, on nous dit plutôt que, à la fois, ils «précipitèrent l'explosion du malaise général et mûrirent l'humeur à la révolte $»^{3}$. Ils marquent donc une accélération brusque dans la chaîne causale déjà donnée et portent à

F. Pardigon, Épisodes des journées de juin 1848, op. cit., p. 86. Pardigon poursuit : "L'homme qui, riche ou pauvre, refuse de sacrifier aux faux dieux, foule sous ses pieds les fétiches de sacristie et d'arrière-boutique, et ose embrasser la statue de la Liberté, ne fait point un pareil acte sans un grand préjudice pour ses intérêts les plus immédiats, pour ses affections les plus chères. Longtemps, il s'en ressentira ».

Ibid. 
point un état d'esprit, en s'inscrivant dans un processus de maturation déjà à l'œuvre. Ils sont donc tout sauf des moments de rupture à effets immédiats, qui provoqueraient, pour ainsi dire instantanément, l'insurrection. Ils précipitent une explosion et mûrissent un état d'esprit. Qu'est-ce à dire ?

Notre hypothèse est la suivante : ces événements ne causent pas l'insurrection, ils déterminent les éléments de la situation à devenir les causes de l'insurrection. On pourrait dire, si la métaphore n'était pas mauvaise, que ces événements créent une atmosphère dans laquelle les éléments objectifs de la situation se cristallisent en causes d'un bouleversement dont, ni les causes ni les deux événements, à eux seuls, ne rendent raison. Pourquoi départager causes et événements ? Pourquoi rapporter les événements à des raisons structurelles larges, à effets médiatisés, et les causes à des raisons conjoncturelles précises, qui semblent à la fois agir immédiatement sur la situation et rester insuffisantes pour la transformer ? «Précipiter » et « mûrir » nous indiquent que, au point où les événements font effet sur l'ordre des causes, un quelque chose advient que la situation ne pouvait déterminer - c'est-à-dire prévoir comme tel, inscrire dans ses possibles - mais à quoi elle laissait une place, ou plutôt une place flottante. Les événements désignent un ordre particulier et original de causes qui affectent cette place flottante, par l'intermédiaire de laquelle les éléments objectifs de la situation se cristallisent en causes de l'insurrection. C'est à la lumière de cette hypothèse que nous devons relire la distinction entre le statut des causes et celui des deux événements dans l'analyse de Marx.

Certes, les « causes » jouent un rôle fondamental. Mais elles ne pourront fonctionner comme telles, comme causes, qu'à certaines conditions dont on pourrait dire qu'elles les activent, ou les constituent rétroactivement comme causes, comme les causes qu'elles auront été. Les causes ménagent la possibilité d'un vide dans l'espace plein et saturé des intérêts sociaux. Mais il doit y avoir quelque chose en plus de ces causes ; il manque quelque chose à cet ordre causal qui articule les intérêts économiques de chacune des 
classes, leurs rapports et la manière dont chaque classe perçoit les autres sous les rapports qu'elle entretient avec elles. Pour que la révolution de Février s'enclenche, il aura fallu que le nexus des causes laisse une marge, un espace vacant, une case vide dont l'investissement cristallisera l'ensemble en « ensemble causal de la révolution de Février ». Voilà comment nous pourrions formuler la manière dont, à l'ordre causal, s'ajoutent (mais précisément pas comme la simple addition d'un facteur isomorphe) des événements, pour produire une insurrection révolutionnaire irréductible à un simple « effet » de ces causes. Il faut que, dans l'espace saturé de sens et d'intérêts objectifs des rapports sociaux (ou plus exactement dans l'espace de leur représentation idéologique), apparaisse une « case vide » qui puisse être investie différentiellement, justement en raison de sa vacuité, par les différents groupes en présence. Ainsi l'aristocratie apparaît en février 1848 comme la classe ennemie de tous, mais différemment pour chacun - c'est-à-dire pour des raisons différentes qui lui donnent, à chaque fois, une signification différente :

«Et les fractions de la bourgeoisie française exclues du pouvoir de crier «Corruption !» Le peuple criait : « À bas les grands voleurs ! À bas les assassins !» quand en 1847 sur les plateaux les plus illustres de la société bourgeoise on jouait publiquement les mêmes scènes qui conduisent régulièrement le prolétariat encanaillé (l'aristocratie financière) dans les bordels, les maisons des pauvres et les asiles d'aliénés, devant les juges, aux bagne et à l'échafaud. La bourgeoisie industrielle voyait ses intérêts menacés, la petite-bourgeoisie était moralement indignée, l'imagination populaire scandalisée, Paris inondé de pamphlets ». ${ }^{1}$

Un même comportement menace les uns, indigne les autres, scandalise d'autres encore. Voilà le sens des « événements»: précipiter l'explosion d'un « malaise », mûrir une « humeur », autrement dit provoquer un investissement affectif commun mais surdéterminé sur la forme où se révélera la contingence de l'ordre

Ibid., p. 14. 
social. La condition d'une insurrection ne peut jamais être qu'un processus fantasmatique, projetant un imaginaire dans une forme vide qui se prête à être surinvestie, c'est-à-dire corrélativement investie par autant de structures fantasmatiques hétérogènes correspondant aux différentes classes en présence. La condition d'une révolution, entendue ici comme refus d'un ordre social, c'est donc le surinvestissement fantasmatique d'une forme vide par les classes en présence, c'est-à-dire sa surdétermination par les différents fantasmes de classe, de telle sorte que cette forme vide puisse servir comme telle de médiation imaginaire dans le processus décisif de radicalisation explosive de la lutte. Rien ne garantit que cette médiation serve plutôt le processus révolutionnaire qu'inversement un processus « réactionnaire ». Effectuant un premier bond dans l'histoire, on prendra pour exemple de case vide coagulant une tendance réactionnaire Louis-Napoléon (et, à l'inverse, Cavaignac et la République bourgeoise de 1848, équivalents ici de l'aristocratie financière en février). À la question : comment se fait-il que Louis-Napoléon parvienne au pouvoir ? La réponse de Marx est presque : en raison de sa vacuité, telle que des classes aux intérêts parfois complètement contradictoires ont pu s'y réunir par le biais d'un investissement fantasmatique.

«L'élection de Napoléon, c'était pour le prolétariat la destitution de Cavaignac, le renversement de la Constituante, le congédiement du républicanisme bourgeois, la cassation de la victoire de Juin. Pour la petite bourgeoisie, Napoléon signifiait le pouvoir du débiteur sur le créancier. Pour la majorité de la grande bourgeoisie, l'élection de Napoléon, c'était la rupture ouverte avec la fraction dont elle avait dû se servir un instant contre la révolution, mais qui lui était devenue insupportable dès qu'elle chercha à consolider la position du moment en position constitutionnelle (...). En votant pour Napoléon, l'armée enfin vota contre la garde mobile, contre l'idylle de la paix, pour la guerre. Il arriva ainsi, comme le constatait la Neue Rheinische Zeitung, que l'homme le plus simple de France acquit la signification la plus complexe. C'est justement parce qu'il n'était rien qu'il 
pouvait tout signifier, sauf lui-même. Cependant, quelque varié que pouvait être le sens du nom de Napoléon dans la bouche des différentes classes, chacune d'elles écrivit ce nom sur son bulletin : "À bas le parti du National, à bas Cavaignac, à bas la Constituante, à bas la République bourgeoise" ». ${ }^{1}$

$\mathrm{Si}$ les événements économiques de portée mondiale sont à prendre en considération, c'est donc dans la mesure où ils suscitent mais pas comme les causes d'un effet - les investissements fantasmatiques d'une case vide et provoquent, de cette manière ou par ce biais différentiel (c'est-à-dire gonflé d'une pluralité de déterminations virtuelles), une insurrection. Pour le dire plus simplement, c'est parce qu'il y eut la maladie de la pomme de terre, plusieurs mauvaises récoltes et la crise générale du commerce et de l'industrie, avec ce que cela implique comme conséquences concrètes sur la vie quotidienne de chacun, que les attitudes, pensées, pratiques de l'aristocratie financière - qu'auparavant le peuple détestait plus ou moins, mais sans que cela débouche sur une insurrection - sont apparues à la population française insupportables. C'est en raison de ces événements que le peuple français investit l'aristocratie comme intolérable, tout en investissant une série de signifiants - « République », « liberté », « émancipation de l'homme », etc. : on y reviendra - dont l'aristocratie financière était devenu le strict opposé. Marx : « Les ravages causés dans le commerce et l'industrie par l'épidémie économique rendirent encore plus insupportable l'autocratie de l'aristocratie financière », au point qu'elle devint intolérable et que son renversement parut évident et nécessaire ${ }^{2}$.

\footnotetext{
Ibid., p. 56-57.

Ibid., p. 15.
}

2 


\section{Problèmes d'inscription : continuité ou rupture?}

Ce développement sur l'analyse marxienne des motifs de l'insurrection de Février visait à montrer une chose : Marx n'applique pas la dialectique à une situation. La dialectique se trouve tordue, pour laisser place à l'écart virtuel qui dessine les lignes de fuites dans l'ordonnancement d'une situation. Nous pouvons y voir un enjeu théorique : celui du sens d'un événement ou de son interprétation, comme problème de l'inscription de l'événement et des modalités de cette inscription, c'est-à-dire de son enregistrement sur tel ou tel ordre, selon telle ou telle logique, constitutive de telle ou telle temporalité. C'est bien le problème du sens de l'événement que celui de son enregistrement ou de son inscription, en ce qu'il s'agit de savoir s'il rompt la rationalité du monde dans lequel il survient, s'il la reconfigure, la bouleverse, ou s'il s'y fond, s'y laisse reprendre et intégrer. Mais c'est bien plus qu'un enjeu théorique : l'enjeu est également pratique, qui apparaît dès les premières heures de la révolution de 1848, et auquel Marx est particulièrement sensible et attentif. Interpréter l'événement, c'est aussi et peut-être surtout le faire. Examinons cela de plus près.

À l'instar de nombre de ses contemporains, Marx voit avant tout dans ce moment insurrectionnel un conflit d'interprétation. Conflit entre ceux qui cherchent à perpétuer ce moment comme tel, dans le geste de rupture qu'il induit ${ }^{1}$, et ceux qui cherchent à le ressaisir comme un simple moment de transition. Conflit entre ceux qui cherchent à inscrire l'événement dans une durée propre, en vue de la recréation du monde, c'est-à-dire de l'organisation politique, sociale et économique - le temps repart à zéro ; et ceux qui cherchent à le réinscrire dans la durée de l'ordre précédent, comme une mutation de

On pourrait dire avec Badiou : ceux qui s'emploient à « tenir » l'événement dans la radicalité de ses conséquences. Sur ce thème, on lira les livres V à VIII de Logique des mondes, ainsi que la longue préface. Voir A. Badiou, Logique des mondes - L'être et l'événement, 2, Paris, Seuil, coll. " L'ordre philosophique », 2006. 
plus ou moins grande importance de cet ordre, se basant par conséquent sur une temporalité dont le rythme doit être de même type que le précédent, à rétablir au plus vite.

Le 25 février constitue sans doute un moment exemplaire de ce conflit. Le Gouvernement provisoire et la délégation ouvrière menée par Raspail sont en conflit. L'enjeu est le suivant : que va-t-on faire de l'insurrection? Les premiers - auxquels s'ajoutent les «lobbies» conservateurs, qui ont beau jeu de faire pression sur un Gouvernement sans majorité légitimée par une quelconque élection souhaitent, le plus vite possible et pour diverses raisons, apaiser les conflits, trouver des compromis qui permettront un retour au calme et par conséquent, c'est là le problème, un retour à l'ordre. Les seconds, les ouvriers et les clubs qui les supportent, refusent quant à eux de se voir reportés à un pré-février, c'est-à-dire à l'ordre qui définissait la Monarchie de Juillet. Peu leur importent les raisons évidentes qui sont invoquées. Car elles sont invoquées sans aucun doute du point de vue du bon sens, c'est-à-dire du point de vue de l'évidence des significations et des partages propres à l'ordre qui vient justement d'être bouleversé. Le bon sens repose sur la présupposition qu'il n'y a que cet ordre, qu'il est dès lors nécessaire de préserver de toute mutation, forcément perçue comme une menace d'anarchie. C'est là une constante, durant toute la conjoncture 18481851 : les conservateurs disqualifient toute contestation de l'ordre comme relevant de l'anarchie et du socialisme, et n'y voient qu'un chaos mortifère, la fin de tout ce qui est et de tout ce qui peut être, la destruction absolue de toute existence. On se reportera sur ce point aux pages que Marx consacre à la période, particulièrement tendue, qui précède les élections complémentaires du 10 mars 1850. On y trouve le bien-nommé « parti de l'ordre » au pouvoir parlementaire, affolé par la manifestation de la moindre différence, du moindre désaccord, qui s'engage dans un délire paranoïaque consistant à recoder toute position contraire à la sienne en termes d'anarchie ou de socialisme, et particulièrement le parti de l'opposition, «ce parti de l'anarchie, comme le baptisent ses adversaires » : 
«Suppression des droits protecteurs - du socialisme ! car c'est s'attaquer au monopole de la fraction industrielle du parti de l'ordre. Réglementation du budget de l'État - du socialisme ! car c'est s'attaquer au monopole de la fraction financière du parti de l'ordre. Libre entrée de viande et de blé étrangers - du socialisme ! car c'est s'attaquer au monopole de la troisième fraction du parti de l'ordre, la grande propriété foncière. Les revendications du parti des freetraders, des libres-échangistes, c'est-à-dire du parti bourgeois anglais le plus progressiste, apparaissent en France comme autant de revendications socialistes. Voltairianisme - du socialisme ! car il s'attaque à la quatrième fraction du parti de l'ordre, les catholiques. Liberté de la presse, droit d'association, instruction publique générale - du socialisme, encore du socialisme! Ils portent atteinte à l'ensemble du monopole du parti de l'ordre ${ }^{1}{ }^{1}$

Mais revenons aux semaines qui suivent l'insurrection de février 1848. Peu importent aux ouvriers les raisons que le bon sens peut invoquer pour ne pas prolonger la crise provoquée par Février ${ }^{2}$. L'essentiel est de tenir le renversement de la Monarchie de Juillet, c'est-à-dire d'accomplir le pas de la rupture avec un monde désormais passé dont les significations ne peuvent plus avoir cours, au profit d'un monde à venir. Ce monde à venir n'est pas un monde imaginaire, préexistant dans le monde antérieur à titre de possibilité politique. C'est au contraire un monde rigoureusement inimaginable. Personne ne pouvait l'imaginer avant Février, plus exactement avant que ne soient tenus les événements de Février dans la radicalité de ce qu'ils impliquent, à savoir le renversement de Juillet et de l'ordre (social, économique, culturel) qui va avec, pour forcer la construction d'un monde nouveau. Pour ceux qui se rangeront bientôt du côté du parti de l'ordre, les transformations provoquées

Ibid., p. 119-120.

2 Par exemple, la nécessité de relancer l'économie au plus vite ou les dangers, non seulement économiques, mais aussi politiques, militaires, etc., que pourrait amener une prolongation de l'indétermination issue de l'insurrection. 
par l'insurrection de février sont des transformations pensables par, dans et sous Juillet, qui auraient même été réalisables sous Juillet si Guizot et Louis-Philippe s'étaient montré plus sympathiques et conciliants. Adopter une telle position revient à refuser le caractère événementiel de Février, au sens très précis où l'insurrection n'y apparaît pas comme un bouleversement radical, c'est-à-dire un véritable pas d'écart du temps lui-même, mais est prise pour une rupture dans le temps et une rupture d'un même temps. La rupture induite par l'événement est par contre un changement de siècle et de monde, une «transformation révolutionnaire du monde » écrit Marx, siècle et monde encore indéterminés, mais gros d'un avenir à construire sur les ruines d'un passé à jamais passé ${ }^{1}$. C'est sans doute ainsi qu'il faut entendre l'énigmatique et célèbre formule du Dix-huit Brumaire de Louis Bonaparte : "La révolution sociale du XIXe siècle ne peut pas tirer sa poésie du passé, mais seulement de l'avenir $\gg^{2}$. Il ne faut pas comprendre que la révolution a pour condition une anticipation prophético-scientifique de ce qui sera (de ce qui doit être) et vers laquelle elle se tendrait, mimant l'avenir « àfaire » au lieu de mimer le passé « déjà-fait », ce qui n'est qu'une autre manière de se rapporter « au passé » en soumettant l'action aux conditions de la récognition de son sens déjà-donné. Par cette formule, il faut plutôt entendre que la révolution s'inscrit dans une temporalité qu'elle pose elle-même : la révolution sociale du XIXe siècle a pour vocation d'autoconstituer sa durée propre. En ce sens, l'action révolutionnaire produit en même temps des actes et la signification de ces actes, au lieu d'agir en fonction de significations déjà données - décidées a priori - et d'interpréter après-coup le sens de ses actions en se demandant s'il faut ou non les assumer.

L'enjeu de la rencontre entre le Gouvernement provisoire et la délégation d'ouvriers est donc : à quelle transformation plus ou moins radicale de l'ordre ancien en un ordre nouveau l'insurrection

Ibid., p. 25.

2 K. Marx, Le Dix-huit Brumaire de Louis Bonaparte, op. cit., p. 122. 
doit-elle conduire ${ }^{1}$ ? Et « le 25 février vers midi », cet enjeu prend la tournure concrète et momentanée suivante : proclamation de la République ou non ${ }^{2}$ ? À cette occasion et pour cette fois - le fait est assez rare dans les événements de 1848 pour qu'on le souligne - le rapport de forces tourne à l'avantage des ouvriers. La République est proclamée. On ne s'étonnera pas qu'alors « s'effaçait jusqu'au souvenir des mobiles et des objectifs limités qui avaient précipité la bourgeoisie dans la révolution de Février $»^{3}$. La radicalité de la transformation va de pair avec une inscription de l'événement dans une durée qui s'auto-détermine - la République était en réalité la cause de la République, on retrouve ce thème chez les blanquistes parce qu'elle construit sa propre surface d'enregistrement : sa propre mémoire.

Que le rapport de force tourne en faveur des ouvriers signifie que, « en imposant la République au Gouvernement provisoire et, à travers celui-ci, à toute la France, le prolétariat se plaçait d'emblée au premier plan comme un parti autonome $»^{4}$. Il est non seulement pour un temps à l'avant de la scène révolutionnaire, mais surtout il n'est soumis à aucun autre parti et ne prend ses décisions que par luimême et pour lui-même. Il est autonome parce qu'il est à l'ouverture $\mathrm{du}$ nouveau monde, l'ouverture républicaine et, à ce titre,

Nous dramatisons de façons outrancière une situation qui, évidemment, était plus compliquée : il faut compter en effet de multiples positions intermédiaires entre ces deux camps, comme par exemple la position de la bourgeoisie républicaine, de la petite-bourgeoisie, à l'intérieur desquelles sans doute se démultiplient les attitudes et positions, presque à l'infini. Reste que, ce que l'événement produit et manifeste dans la rupture qu'il provoque, c'est une radicalisation, aux yeux de ses acteurs, des positions entre ces deux attitudes extrêmes. En effet, vues sous l'angle de cette opposition duelle radicale, les positions intermédiaires apparaissent comme autant de tentatives de « changer mais pas trop quand même », c'est-à-dire, en définitive de n'effectuer de changement que dans certaines limites fixées par l'ordre précédent, auxquelles le bon sens impose de ne pas toucher.

2 K. Marx, Les luttes de classes en France, op. cit., p. 17.

3 Ibid., p. 18 (nous soulignons).

4

Ibid. 
potentiellement socialiste. Sans doute cette visibilité et cette autonomie doublée de solitude lui coûteront-t-elles très cher en juin. Reste que, malgré les risques effectifs qu'elles comportaient, ce ne fût pas peine perdue pour le prolétariat : «ce qu'il conquit, c'était le terrain en vue de la lutte pour sa propre émancipation révolutionnaire ${ }^{1}$. L'émancipation devient possible grâce au terrain qui fait droit, au grand jour, à certaines revendications auparavant secrètes, le terrain qui permet de poser devant tous certains enjeux autrefois objets de conspirations cachées dans les coulisses de la scène politique. Si nous insistons sur ce conflit qui tourne à la faveur des ouvriers, actifs et autonomes, c'est pour marquer combien l'insurrection de Février n'est pas la conjonction de la mystification bourgeoise et de la stupidité de la classe ouvrière, incapable de comprendre que la réalité économique l'empêchait de faire de cette révolution une vraie révolution. En clair : cette insurrection n'est pas une insurrection de surface ; ce n'est pas la répétition grotesque des grands événements de 1789 dans le théâtre parlementaire, ni un semblant d'action incapable d'ouvrir l'histoire à sa vérité. À même l'événement, Marx pointe bien les virtualités révolutionnaires à l'œuvre - bien qu'elles soient toujours également susceptibles d'être réinterprétées comme autant de degrés de progression sur une ligne du temps prédéterminée : avancement de la révolution comme vérité de l'histoire vers son avènement. Or, ce faisant, se révèle la possibilité d'une autre rationalité de l'événement révolutionnaire, un autre mode de compréhension de ses «réussites» et de ses « échecs ». C'est ce qui apparaît dans l'analyse du retour de flamme que subit la classe ouvrière en mai-juin 1848 .

Ibid. 


\section{De la victoire à l'échec, de la défaite à la victoire : questions de mots}

Très vite, après la proclamation de la République, le rapport de forces s'inverse. Ce qui fut conquis n'était en effet pas encore la révolution, mais son terrain, sa scène, ses acteurs costumés de slogans et attifés de mots d'ordre. Ainsi républicains bourgeois et démocrates du gouvernement provisoires acceptent-ils certaines revendications ouvrières. Les signifiants «République », « liberté » ou « émancipation» ainsi que « organisation du travail », « ateliers sociaux », « institutions sociales » ou « ministère du travail » firent la victoire de Février. Mais, pour l'essentiel, ils resteront des mots. Marx l'écrit en toutes lettres :

« Tout comme la monarchie de Juillet fut contrainte de proclamer qu'elle était une monarchie entourée d'institutions républicaines, la république de Février fut forcée de s'annoncer comme une république entourée d'institutions sociales. Le prolétariat parisien arracha aussi cette concession ${ }^{1}{ }^{1}$

S'annoncer, écrit Marx : il s'agit bien de mots, et de rien d'autre. Le gouvernement provisoire s'emploiera à faire de cette annonce un vœu pieu, d'abord par le relatif ridicule des institutions en question au regard des revendications ouvrières (par exemple les ateliers nationaux plutôt que les ateliers sociaux, en y rejouant, victorieusement cette fois, la lutte linguistique), puis par leur progressive suppression (ainsi la suppression, petit à petit, des ateliers nationaux, de mai à juillet 1848).

Ceci semble bien confirmer l'hypothèse canonique selon laquelle Marx n'aurait vu dans la révolution de Février qu'un changement superficiel, permettant seulement d'épuiser une forme idéologique de plus, avant d'en venir au contact de la réalité sociale et économique. Pour filer la métaphore : un changement de costumes n'affectant pas les corps. Mais la métaphore est mauvaise, à rester

Ibid., p. 19. Nous soulignons « s'annoncer». 
aussi pauvre. S'il n'y a pas encore de mise à nu de la réalité dans la révolution de 1848, cela ne veut pas dire qu'il n'y a pas de changement. Il y a changement de costumes, c'est-à-dire également changement de la perception que chacun a de lui-même et d'autrui, changement des rapports entre les corps, et des attitudes que ces corps adoptent. Autrement dit, s'il est vrai que Marx voit dans la révolution de Février avant tout une révolution du discours - d'où tout le vocabulaire théâtral du costume et de la scène qui traverse Les luttes de classes en France et qui sera repris dans Le Dix-huit Brumaire -, on ne peut pour autant en conclure que rien ne se soit passé, ni que les ouvriers se soient purement et simplement laissé berner. Nous touchons ici à un point particulièrement important de l'analyse marxienne de 1848. Si les enjeux et les mots d'ordre révolutionnaires peuvent devenir cela, « juste des mots », "paroles seulement », c'est précisément qu'ils l'étaient dès le départ, toujours déjà ; c'est précisément parce que depuis le départ on ne se battait que pour des mots et par rapport à des mots, pas pour des intérêts, des raisons, des motifs socio-économiques objectifs - ce qui ne veut pas dire que ces derniers n'existaient pas mais, comme on l'a vu, qu'ils n'auraient pu produire à eux seuls la précipitation du processus insurrectionnel. Les mécanismes de précipitation et de maturation, que nous avons pris soin de distinguer de mécanismes causaux, parce qu'ils transforment les conditions en causes, s'ordonnent d'abord à des signifiants-en-souffrance (« liberté-égalité-fraternité », « république », « république sociale », « organisation du travail », " reprendre la révolution là où le 9 thermidor l'a arrêtée », etc.). Dans de tels signifiants peuvent se projeter des désirs échappant au système des intérêts propre à la reproduction de l'ordre social, donc des désirs qui ne savent pas encore eux-mêmes ce qu'ils sont, ce qu'ils veulent, où ils vont, des désirs qui sont contraints de définir leur « objet » à mesure qu'ils se construisent eux-mêmes en fonction d'une « pulsion » subjective et non d'une finalité préalablement donnée ou conçue.

Pour autant, ces signifiants ne sont pas neutres : ils déterminent une redistribution des enjeux politiques. Qu'on observe la 
description donnée par Marx des activités principales du Gouvernement provisoire, de février à juin. Une fois proclamée la République, l'essentiel pour le Gouvernement provisoire, à dominante bourgeoise ${ }^{1}$, c'est de « la rendre acceptable à la bourgeoisie et aux provinces $\gg^{2}$. Programme idéologique aux enjeux - et aux effets - bel et bien réels. Il s'agit certes de réduire la République à de simples mots, mais pas à n'importe lesquels. Ainsi du refus de proclamer la République sociale, ainsi de la création d'ateliers nationaux plutôt que sociaux, ainsi encore de la proclamation de la « nature pacifique » de la république « aux puissances despotiques de l'extérieur $»^{3}$. Lutte discursive qui atteint son acmé lors de la première réunion de l'Assemblée constituante, le 4 mai 1848. Marx y insiste : la République,

« ce n'est pas la République imposée au Gouvernement provisoire par le prolétariat parisien, ce n'est pas la République pourvue d'institutions sociales, non pas l'image de rêve que caressaient les combattants des barricades. La République proclamée par l'Assemblée Nationale, la seule légitime, c'est la République qui n'est pas une arme révolutionnaire contre l'ordre bourgeois, mais plutôt sa reconstitution politique, la consolidation politique de la société bourgeoise, en un mot : la République bourgeoise ${ }^{4}{ }^{4}$

Est à l'œuvre - dans ce pas d'écart de la bourgeoisie par rapport à un ordre antérieur, qui lui apparaît maintenant, rétroactivement, comme étant l'ordre qui seul peut satisfaire ses intérêts - la volonté

1 Le Gouvernement provisoire comportait seulement deux représentants des ouvriers, Louis Blanc et Albert, aucun d'entre eux n'étant ministre, puisque le premier avait été relégué à la présidence de la Commission de réflexion sur le travail dite «Commission du Luxembourg », et que le second n'avait aucune tâche à proprement parler - de sorte qu'ils n'étaient là aux yeux des bourgeois du Gouvernement qu'à titre de représentant des ouvriers, "parce qu'il faut bien ».

Ibid., p. 24.

Ibid.

$4 \quad$ Ibid., p. 36. 
de la bourgeoisie de réinscrire les événements dans cet ordre et de déterminer en conséquence les mots d'ordre de l'insurrection. Dès lors, la tâche de la République « cessa d'être la transformation révolutionnaire du monde ; elle consista uniquement à s'adapter aux conditions de la société bourgeoise ${ }^{1}$.

Est-ce là un simple retour ? Lorsque Marx nous dit que « les républicains bourgeois du National s'amusèrent à substituer aux noms et costumes monarchiques ceux de l'ancienne République », que « pour eux, la République n'était qu'une nouvelle toilette de bal pour la vieille société bourgeoise $»^{2}$, retombons-nous dans l'interprétation selon laquelle les ouvriers furent mystifiés par la bourgeoisie, bernés par sa rhétorique ? Pas du tout. Précisément parce que pour les ouvriers, la République n'est pas une nouvelle toilette de bal. Elle doit mener à une République sociale, et celle-ci, à son tour, vers un monde à construire. Autrement dit, la lutte pour et par des mots a ceci d'essentiel qu'elle oriente, en tant que lutte, c'està-dire en fonction des investissements désirants qui la portent, leur détermination et le codage de ces investissements en tel ou tel ordre social. Néanmoins, on retrouve ici la marque dialectique de l'analyse de Marx. Là où des conspirateurs comme Blanqui prennent la vacuité des mots d'ordre, leur indétermination, comme l'occasion d'une élaboration d'un nouveau monde, c'est-à-dire d'un monde dont le contenu, les partages, l'organisation restent indéterminés, Marx tend à rabattre cette vacuité sur une double ignorance de la classe ouvrière : une ignorance de ce qu'il faut faire ; une ignorance de la situation actuelle. Cette ignorance de la situation actuelle qui aurait empêché la réussite de l'insurrection précisément pour les mêmes raisons qui l'avaient provoquée : parce que les mots d'ordre restaient vides, et ne trouvaient pas leur contenu propre. Ignorance, et donc incapacité : « Nous avons vu que (...) le prolétariat parisien était encore incapable d'avancer au-delà de la République bourgeoisie autrement qu'en idée, qu'en imagination, que partout où il passait

Ibid., p. 25.

2 Ibid., p. 24. 
vraiment à l'action, il agissait au service de celle-ci » ${ }^{1}$. Si les mots ne sont pas nécessairement synonymes d'illusions idéologiques, il reste qu'ils ne suffisent pas à faire de la lutte une lutte réelle, lorsqu'ils restent vides de référents déterminés.

Or - c'est là une des forces théoriques du texte de Marx - cette ignorance qui condamne la classe ouvrière à l'échec est également ce qui transforme cette expérience en réussite, parce qu'elle dégrise l'ivresse verbale du prolétariat et lui manifeste les raisons pour lesquelles il a échoué ainsi que, en creux, les raisons qui le mèneront, in fine, à la victoire. Nous reviendrons sur ce point, indice d'une véritable théorie de l'apprentissage chez Marx, apprentissage de la révolution et de ses conditions, qui fait de ses analyses de 1848 un Bildungsroman du prolétariat. Relevons pour l'instant que la lutte de février à juin autour de la détermination du signifiant « République », bien que perdue par les ouvriers dans l'immédiat, est néanmoins le signe d'une victoire, dans la mesure où elle permît une clarification du partage des classes en luttes, des alliances et ruptures durant les mois suivants. Cette clarification, Marx l'interprète dans les termes d'une loi historique célèbre : la simplification tendancielle des antagonismes sociaux. Il convient de l'examiner de plus près, car nous touchons ici au point nodal dans Les luttes de classes en France, à savoir l'enjeu capital, aussi bien pratique que théorique, de l'inscription de l'événement.

\section{Dialectique et démystification}

Rappelons la thèse de la simplification des antagonismes de classes, énoncée dans le Manifeste :

«La société bourgeoise moderne, qui est issue des ruines de la société féodale, n'a pas surmonté les antagonismes de classes. Elle a mis seulement en place des classes nouvelles, de nouvelles conditions d'oppression, de nouvelles formes de luttes à la place des anciennes. Toutefois, notre époque -

Ibid., p. 36 . 
l'époque de la bourgeoisie - se distingue par un trait particulier : elle a simplifié les antagonismes de classes. De plus en plus, la société se divise en deux grands camps ennemis, en deux grandes classes qui s'affrontent directement : la bourgeoisie et le prolétariat ». ${ }^{1}$

Cette thèse a d'abord une valeur descriptive : elle décrit le mouvement de l'histoire sociale. Mais c'est trop peu dire. Car Marx lui imprime une détermination dialectique. Il en résulte un double effet : d'une part, ce mouvement est un mouvement nécessaire ; d'autre part ce mouvement d'opposition est un mouvement contradictoire qui, dans sa radicalité la plus grande, au point même de sa radicalité absolue, se retourne pour être relevé, dans l'Aufhebung d'une société sans classes. Sur cette base, Les luttes de classes en France tend à recoder l'événement de Février et ses conséquences dans le sens de cette dialectique, c'est-à-dire à leur assigner une place sur ce mouvement, en fonction de la maturation $\mathrm{du}$ processus historique. Ce qui pose à Marx le problème suivant, crucial pour la pratique révolutionnaire : comment évaluer le degré de maturation du processus à un moment donné ?

La réponse est dans la question : en rapportant le processus à son point terminal d'aboutissement, celui de l'antagonisme poussé à son point le plus aigu, «bourgeoisie versus prolétariat », qui servira donc de critérium. Il permet de repérer le point de maturation de l'événement, c'est-à-dire de l'inscrire sur l'échelle des degrés par lesquelles passe le mouvement dialectique de simplification des antagonismes. Et c'est bien à partir de l'axe « Bourgeoisie industrielle versus Prolétariat » que Marx mesure le progrès apporté par Février. Renversant les représentants de l'aristocratie financière, l'insurrection de février va permettre, au fil de détours dont il serait trop long de rappeler toute la complexité ici, de porter la bourgeoisie industrielle au sommet de la République, avec l'aristocratie

K. Marx et F. Engels, Le Manifeste communiste, dans Karl Marx, Euvres I, op. cit., p. 162. 
financière ${ }^{1}$. Autrement dit, au pouvoir, nous trouvons désormais la bourgeoisie dans son ensemble, ou plus exactement dans sa pureté, puisque la petite-bourgeoisie se voit progressivement rejetée parmi les dominés, aux côtés du prolétariat, en tant qu'elle s'oppose à l'aristocratie financière ${ }^{2}$. On voit bien opérer ici la simplification progressive des antagonismes, entre deux camps - bourgeois versus petits-bourgeois et prolétaires - qui se forment peu à peu suite à Février. Désormais la scène opposera donc la bourgeoisie dans sa pureté de classe à ceux qu'elle domine et ruine progressivement (petits-bourgeois, paysans et même bourgeois républicains), voués petit à petit à se rassembler autour du prolétariat, sinon à se fondre en lui au fur et à mesure de la simplification qui tend à ne laisser plus que deux classes en vis-à-vis. Pour résumer d'une formule : les luttes de classes (multiples) se transforment en la lutte des (deux) classes.

L'échec de la révolution de Février devient alors compréhensible et, mieux encore, acceptable, dans la mesure où les raisons de son échec sont les mêmes que celles de sa réussite relative : sa place sur l'échelle de la simplification. Si la révolution de Février échoue, c'est bien parce qu'elle n'est qu'un moment de cette simplification. La complexité de la situation politique et économique (la place ambiguë de la bourgeoisie industrielle, l'importance numérique relativement faible du prolétariat par rapport à une population majoritairement rurale, etc.) ne permettait pas l'explosion véritablement révolutionnaire, celle de l'ordre bourgeois en tant que tel. Littéralement : la situation n'était pas assez simple. Si cette révolution n'a pas mené à la révolution véritable directement, si elle doit passer par tous ces détours qui la définissent, c'est donc pour les mêmes raisons qui font qu'elle doit y mener indirectement.

Voir K. Marx, Les luttes de classes en France, op. cit., p. 104 : « Toute notre analyse a montré que la République, depuis le premier jour de son existence, n'a pas renversé l'aristocratie financière, mais l'a consolidée ».

2 Voir ibid., p. 119 : « Nous avons vu peu à peu paysans, petits-bourgeois, les classes moyennes en général, passer aux côtés du prolétariat, forcés de s'opposer ouvertement à la République officielle, traités en adversaires par celle-ci ». 
Advenue dans une situation trop complexe encore, elle prépare la radicalisation simplificatrice des antagonismes en un antagonisme. C'est dire que, selon Marx, il s'agit bien plus que d'une simple clarification de la situation : il s'agit bel et bien de son évolution, à défaut de sa révolution - d'une rupture temporelle la rejetant à jamais au passé.

En quoi consiste cette évolution ? Avant tout dans une démystification. L'incipit du premier article l'annonçait :

«Ce qui succomba dans ces défaites, ce n'est pas la révolution. Ce sont les traditionnels accessoires prérévolutionnaires, résultant de conditions sociales qui ne s'étaient pas encore exacerbées en conflits de classes personnes, illusions, projets, dont le parti révolutionnaire n'était pas libéré avant la révolution de Février, dont il ne pouvait être libéré par la victoire de Février, mais seulement par une série de défaites $»{ }^{1}$

Démystification qui, alignée sur la loi de la simplification tendancielle, dessine la ligne directrice de la maturation du processus historique, celle de l'éducation de la classe ouvrière à la lutte et aux moyens de la lutte. De Février, le prolétariat sort grandi, bien que défait. Et cet apprentissage passe avant tout par une démystification d'ordre terminologique, comme le montre la lutte discursive qui s'engage suite au soulèvement de Février. L'insistance de la bourgeoisie au pouvoir (le Gouvernement provisoire, en l'occurrence) à réduire la République à de simples mots conduit le peuple à exiger plus que la seule République, et même autre chose qu'une République, qu'il convient désormais d'appeler systématiquement, selon Marx, «République bourgeoise »². Contre

Ibid., p. 10 .

2 Voir ibid., p. 36. Cette précision lexicale entérinée par les événements de Juin est décisive, car l'ennemi possède désormais un nom : «Après Juin, révolution voulait dire : renversement de la société bourgeoise, tandis qu'avant Février cela avait signifié : renversement du système de gouvernement (Staatsform) », ibid., p. 42. 
« la République des riches ${ }^{1}$, on réclame peu à peu une "République social-démocrate », et même une "République rouge $»^{2}$. L'apprentissage n'est pas le fait des seuls ouvriers. La bourgeoise aussi s'éduque en se trouvant aux prises avec le prolétariat. La concession aux ouvriers de la proclamation de la République entraîne de fait une radicalisation de l'ordre bourgeois ${ }^{3}$ : " surprise, l'Europe se réveilla brutalement de sa torpeur bourgeoise $»^{4}$. Sortie de sa léthargie, la bourgeoisie réagit pour assurer ses conditions d'existence. Les revendications ouvrières apparaissent désormais au grand jour. Comme l'écrit Marx, le « secret de la révolution du XIXe siècle » est « révélé du haut d'une tribune européenne »: l'émancipation du prolétariat, porté par des " "rêves insensés" qui, jusqu'alors enfouis dans les écrits apocryphes des socialistes, ne parvenaient que sporadiquement aux oreilles de la bourgeoisie $»^{5}$. Désormais le mot d'ordre est énoncé « officiellement $»^{6}$. Voilà pourquoi la bourgeoisie tente de réduire la République a un simple mot. Il y a un double renforcement, une double révélation, une double clarification des deux forces principales l'une par l'autre, créant ainsi une circularité révolutionnaire. La dénégation de l'événement par la bourgeoisie, par sa réduction à de simples mots et donc, du même coup, à un fait parmi d'autres dans le cours de l'ordre ancien provoque un durcissement de la situation et une radicalisation de la lutte. Laquelle, à son tour, force la bourgeoisie à réagir plus durement encore, et ainsi de suite. En somme, non seulement la bourgeoisie reconnaît l'événement par sa négation elle-même, mais aussi elle prépare sa pleine et entière réalisation en s'employant à le réduire à

Ibid., p. 56.

2 Ibid., p. 115.

3 Voir ibid., p. 25 à 30.

4 Ibid., p. 23.

5 Ibid.

6 Ibid. 
néant : car, loin de rester identique, le réel en question en vient à changer lui aussi, l'ordre qui auparavant le définissait se trouve renforcé et radicalisé, et avec lui les conditions d'existence de la classe bourgeoise comme telle, de sa domination de classe toute entière, c'est-à-dire, en vis-à-vis, les conditions d'existence du prolétariat, et même plus encore les conditions d'existence de toutes les classes, de tout le peuple français qui est engagé, à vitesses variées et à degrés divers, dans un devenir-prolétaire.

\section{Telos et croyance : le sens des actes}

Ici, l'analyse marxienne penche décisivement vers une forme de « nécessitarisme théoriciste ». Il faut attendre - c'est une question de temps et de patience - que les étapes nécessaires à la constitution de la situation proprement révolutionnaire soient toutes franchies. Mais nous nous trouvons ici devant un problème métaphysique : y a-t-il du proprement révolutionnaire ? Dit autrement : y a-t-il du proprement prolétaire d'un côté, du proprement bourgeois de l'autre, c'est-à-dire des classes pures ? On peut dire que, en inscrivant l'événement sur l'échelle d'un mouvement nécessaire dont l'idéal indéfiniment à venir fixerait l'ordre de la progression, on tend à renverser une certaine conception de la lutte des classes, selon laquelle la lutte est constitutive des classes. Le renversement consiste en ceci : ce n'est pas la lutte qui constitue les classes, mais les classes qui constituent la lutte, parce qu'elles lui préexistent au moins en droit. Ainsi peuvent-elles être définies depuis un rapport simple d'opposition, lequel rapport ne transforme pas, par des échanges et des nœuds complexes, les classes en question, mais au contraire les fixe, les délimite et les définit dans leur pureté de droit. Si bien que l'on peut alors donner l'orientation de la lutte elle-même, indexée sur le mot d'ordre de l'émancipation des ouvriers comme condition de l'émancipation de l'homme. Tout se passe comme si le mot d'ordre « émancipation de l'homme » caractérisait effectivement l'essence de l'histoire et fournissait le telos au regard duquel la prématuration et 
le retard de la réalisation du mot d'ordre pouvaient être évalués dans les différentes conjonctures. L'ensemble des présupposés fait système : il faut (a) rapporter l'histoire humaine à sa "vérité ", qui lui donne en même temps son unité de sens, pour (b) se figurer son telos et lui donner un contenu déterminé, ce qui (c) permet de définir - rôle de Lascience, personnage conceptuel de cette tragi-comédie les étapes nécessaires par lesquelles l'histoire doit en passer avant de réaliser ledit telos, et de la sorte (d) autorise la production d'un diagnostic de la conjoncture qui évalue les degrés relatifs d'avancement et de retard des mouvements réels.

Or ceci soumet à nouveau le temps révolutionnaire aux conditions de la ré-cognition. Voilà pourquoi, en vertu d'une autre tendance interne au texte marxien qui fait pour beaucoup l'originalité des Luttes de classes en France, il semble pertinent d'insister sur ce point : le mot d'ordre « émancipation de l'homme » ne veut rien dire. Nous ne savons pas ce qu'il peut vouloir dire, ou plus précisément : ce qu'il est susceptible d'être amené à avoir voulu dire. Et il n'est pas nécessaire, au moment même où on le crie et se bat en son nom, de savoir ce qu'il veut dire ou pourra vouloir dire - ce qui ne signifie pas, on l'a vu, que le choix des mots d'ordre soit dénué d'importance. Réponse à faire à tous ceux qui affirment que les mots d'ordre révolutionnaire sont vides : oui, et alors ? Ce vide est une condition constituante de l'action révolutionnaire. Mieux, c'est à cette condition qu'il peut y avoir acte et décision en général, et c'est ce qu'il faut justement apprendre à assumer. Non, nul ne sait - et Marx encore moins, Marx le dernier - « comment-on-vivra-dans-lasociété-communiste ». Il n'y a pas lieu de s'en lamenter : c'est parce que nous ne le savons pas que nous la désirons ${ }^{1}$.

Blanqui est éclairant sur ce point - et Marx ne se prive pas de relever son importance, en le comptant parmi «les véritables chefs

Au double sens de cette expression : qu'elle ne soit pas déjà et toujours déjà reconnaissable dans les schèmes dont nous disposons et qui nous sont faits par la société que nous refusons, c'est bien ce qui nous la rend désirable ; et : on ne peut désirer que des signifiants, sinon vides, du moins « insaturés ». 
du parti prolétarien », précisément, croyons-nous, pour cette raison ${ }^{1}$. Dans sa «Lettre à Maillard » ${ }^{2}$, après avoir exprimé à ce dernier son désaccord quant aux causes de l'avortement de Février - et avoir notamment innocenté les philosophes ${ }^{3}$ et les chefs d'école, dont la diversité des opinions n'est pas un ferment de discorde mais un symptôme positif - Blanqui a cette affirmation étonnante, si l'on y regarde d'assez près :

« Le socialisme, c'est la croyance à l'ordre nouveau qui doit sortir du creuset de ces doctrines (...) et déjà, de leurs efforts, il est sorti une résultante qui, sans être encore bien déterminée, a cependant saisi l'esprit des masses, est devenue leur foi, leur espérance, leur étendard ». ${ }^{4}$

Voici donc une définition du socialisme. Mais elle est bien curieuse. Le socialisme est une croyance, d'abord. Traduction : ce n'est point une théorie pleinement constituée, dont la validité nous serait assurée par les moyens de la science ; c'est un acte de foi. Cette croyance porte sur un ordre social, ce qui n'est pas étonnant, mais sur un ordre social à venir. Il ne s'agit donc pas de croire ceci ou cela quant à l'ordre social existant, mais d'engager sa foi dans un ordre qui pourra résulter du mouvement de transformation qu'il s'agit de porter à son terme. Encore cet ordre nouveau doit-il sortir du creuset des doctrines socialistes - des «diverses théories sociales qui se proposent de reconstituer le monde sur la base de la justice et de l'égalité »- dont les efforts n'ont pour le moment abouti à rien de déterminé. Donc, en un mot, le socialisme c'est la croyance à l'ordre

\footnotetext{
$1 \quad$ K. Marx, Le Dix-huit Brumaire de Louis Bonaparte, op. cit., p. 129.

2 Dans A. Blanqui, Maintenant, il faut des armes !, Paris, La Fabrique, 2006. Fondateur d'un Club républicain du $\mathrm{V}^{\mathrm{e}}$ durant les premiers mois de la Révolution de Février, Maillard avait aussi été proche par la suite du pacifisme de Buchez (spiritualiste notoire) et Leroux ; puis fut transporté en Afrique suite aux journées de Juin - de ces différents facteurs découle sans doute l'ambivalence du discours de Blanqui à son égard.

3 Voir ibid., p. 173 : « c'est maintenant plus que jamais qu'il faut être philosophe ». Ibid., p. 173-174.
} 
social qui résultera des doctrines socialistes... Définition purement réflexive, sinon tautologique : le socialisme consiste dans l'acte de foi envers le socialisme - ou : dans l'acte de foi des socialistes envers les socialistes. Ce qui jusqu'à présent en est sorti n'est certes pas « encore bien déterminé », mais sa valeur de vérité ou sa précision ne sont rien eu égard à son efficacité, qui consiste à s'emparer de l'esprit des masses pour devenir leur foi. C'est bien que, du point de vue de son contenu sémantique et théorique, le socialisme n'est rien. Il n'est pas un ensemble de contenus de pensée en vue de la connaissance. Il coïncide avec le mouvement révolutionnaire, il est la forme réfléchie que prend la foi dans l'acte révolutionnaire. « $\mathrm{Ne}$ vous y trompez pas, le socialisme, c'est la révolution. Elle n'est que là ». Ce que Marx affirmera de son côté, évoquant Blanqui, autour du motif de la «déclaration », pour l'opposer au «socialisme doctrinaire » :

«Ainsi, tandis que l'utopie, le socialisme, qui subordonne le mouvement d'ensemble à l'un de ses éléments, qui substitue à la production collective, sociale, l'élucubration du pédant individuel et qui, surtout, escamote en imagination la lutte révolutionnaire des classes, avec ses inexorables exigences, au moyen de petits artifices ou d'un gros sentimentalisme, tandis que ce socialisme doctrinaire, qui, au fond, ne fait qu'idéaliser la société actuelle, en dessine une image sans ombres et voudrait imposer son idéal contre la société existante, tandis que le prolétariat abandonne ce socialisme à la petite bourgeoisie, tandis que les luttes intestines des différents chefs socialistes font ressortir chacun des prétendus systèmes comme l'adoption prétentieuse de l'un des points de passage du bouleversement social au détriment des autres - le prolétariat se groupe de plus en plus autour du socialisme révolutionnaire, autour du communisme, pour lequel la bourgeoisie elle-même a inventé le nom de Blanqui. Ce socialisme est la déclaration de la révolution en permanence, la dictature de classe du prolétariat comme point de transition nécessaire vers l'abolition des différences de classes tout court, vers l'abolition de tous les rapports de production sur lesquels elles reposent, vers l'abolition de 
toutes les relations sociales qui correspondent à ces rapports de production, vers le bouleversement de toutes les idées qui naissent de ces relations sociales $\gg .{ }^{1}$

Définition purement négative du socialisme, sinon par l'acte qui y préside, comme déclaration. Marx est ici au plus proche de Blanqui. Ils se rejoignent dans l'axiome suivant : sans déclaration de foi aveugle, le mouvement s'éteint ; mais on pourrait dire aussi bien, sans le mouvement révolutionnaire, le socialisme est vide - il est le mot d'ordre vide dont le mouvement a besoin pour croire en luimême.

\section{Accélération du temps : l'histoire en train de se faire}

Il ne nous est donc pas permis de trancher sur la perspective de Marx dans Les luttes de classes en France. Celle-ci est irréductiblement double, mieux : divisée en deux tendances incompatibles et contradictoires. Nous les avons vues se succéder. Mais elles se trouvent co-impliquées dans un passage du troisième article qui constitue l'acmé de l'analyse marxienne : les pages consacrées à la période de janvier-mars 1850 , qui voit le retour sur la scène politique d'une coalition rouge forte. Ce n'est pas un hasard. Marx publie son article en avril 1850, sous le titre "Conséquences du 13 Juin 49 ». Il l'écrit donc pendant et juste après l'événement, sans en connaître l'issue.

Marx relève trois éléments. Premièrement, les diverses fractions dominées - principalement le «prolétariat révolutionnaire », " la petite bourgeoisie socialiste » et le «parti bourgeois républicain »composent une «coalition générale contre la bourgeoisie et le gouvernement comme en Février. Mais cette fois, le prolétariat était à la tête de la ligue révolutionnaire $»^{2}$. Conséquence de l'application

K. Marx, Les luttes de classes en France, op. cit., p. 122.

2

Ibid., p. 124. 
rigoureuse de la loi de simplification tendancielle des antagonismes : le rapport de forces s'est inversé avec le durcissement de l'ordre bourgeois sous le parti de l'ordre et Bonaparte. Désormais la scène est claire, c'est l'émancipation du prolétariat qui est le but par lequel l'émancipation de tous est possible. Or, cette coalition triomphe aux élections : «Le parti de l'ordre fut comme foudroyé » ${ }^{1}$. Et Marx de conclure ainsi une tirade qui reprend les trois échecs subis par la gauche depuis deux ans : «Le 10 mars était une révolution $»^{2}$. Marx se paierait-il notre tête ? Une élection, une révolution ? Une élection partielle de surcroit, une élection qui est organisée pour remplacer les députés de la Montagne pourchassés le 13 juin de l'année précédente ! Voilà trois candidats du rose-orange au rouge vif qui remportent trois sièges à Paris, et c'est la révolution ? C'est que, selon Marx, il y a là une virtualité politique révolutionnaire. «Derrière les bulletins de vote, il y a les pavés » ${ }^{3}$. Il y a les pavés parce que - deuxième élément relevé par Marx - le parti de l'ordre s'affole et, comme dit Marx, « revient naturellement à son inévitable lieu commun. "Davantage de répression ! s'écrie-t-il, répression décuplée !" » ${ }^{4}$. Sauf que «sa force de répression est devenue dix fois plus faible, tandis que la résistance a centuplé $»^{5}$. Sont en effet passées du côté du prolétariat toutes les classes intermédiaires avec lesquelles, en juin 48, la bourgeoisie avait pu réprimer l'insurrection ouvrière. Troisième élément, l'affolement du parti de l'ordre. La bourgeoisie procède à un suicide politique en voulant supprimer la République constitutionnelle et le suffrage universel qui, à ses yeux, la menace, alors qu'en réalité - c'est du moins ce que Marx a mis en évidence - la République est la forme sous laquelle la bourgeoisie peut vivre et imposer son ordre sans être prise dans des querelles à la fois politiques (entre orléanistes et légitimistes) et économiques

\footnotetext{
Ibid., p. 125.

Ibid.

Ibid.

Ibid., p. 126-127.

Ibid., p. 127.
} 
(entre grands propriétaires, industriels, financiers). Suicide résumé par Marx comme suit :

« Les fractions coalisées de la bourgeoisie sont déjà condamnées du fait même qu'elles désertent la seule forme possible de leur pouvoir commun, la forme la plus puissante et la plus complète de leur domination de classe, la République constitutionnelle, et retournent à cette forme inférieure, incomplète et plus faible qu'est la monarchie ». ${ }^{1}$

En somme, Marx voit dans cette situation de mars 1850 la révolution à portée de main. Et pourtant, les conditions ne sont pas remplies. L'époque n'est pas mûre. La simplification n'est pas accomplie. Certes, il y a, dans une certaine mesure, simplification. Mais justement, dans une certaine mesure seulement, c'est-à-dire dans la mesure où le degré atteint par la simplification en mars 1850 marque un état plus avancé du processus qu'en février 1848, et donc aussi, du même coup, trop faible encore pour satisfaire aux conditions nécessaires à la révolution, à savoir une simplification radicale. Et en effet, à rigoureusement parler, c'est-à-dire à vouloir trouver dans la situation une fusion parfaite - propre, pure - des classes intermédiaires dans la classe prolétarienne, on risque d'être déçu : il n'y a là qu'une coalition, «coalition d'intérêts divergents » - pas moins d'ailleurs que le parti de l'ordre - qui fait du parti rouge un «soi-disant parti $»^{2}$. D'où l'étrangeté apparente de ces lignes qui suivent l'apparente « démonstration » de la réalisation de la simplification des antagonismes et qui rappellent posément et ironiquement, comme en aparté, les différences idéologiques de la coalition $^{3}$. Reste que Marx se voit devant une révolution qui s'esquisse, dont la raison semble vide, c'est-à-dire non pas déterminée et orientée par des causes communes précises ou par un contenu désiré, mais portée par une coalition multiforme, dont l'unité est, elle aussi, pour le moins vide : « se présenter hautement comme

\footnotetext{
Ibid., p. 128-129.

Ibid., p. 119.

Cf. ibid., p. 121.
} 
moyen d'émancipation du prolétariat et présenter l'émancipation de celui-ci comme son but $»^{1}$. Et Marx détaille le surinvestissement du mot d'ordre : «Tromperie voulue de la part des uns, aveuglement volontaire des autres, qui prétendent que le monde transformé selon leurs propres besoins est le meilleur monde pour tous, la réalisation de toutes les aspirations révolutionnaires et l'abolition de toutes les collisions révolutionnaires $»^{2}$.

Comment Marx parvient-il à se sortir de cette situation, c'est-àdire à la penser ? Par un usage de la thèse de la simplification qu'on pourrait dire, pour reprendre des termes kantiens, "réfléchissant ». Elle permet à Marx de s'orienter dans l'enchevêtrement complexe et mouvant de l'expérience - les rapports multiples de classes multiples. La thèse n'est plus l'expression discursive du mouvement dialectique de l'histoire, c'est une Idée dont la réalisation dépasse l'expérience et qui sert de principe régulateur pour donner un sens à l'expérience, toujours fragile et provisoire, à remettre en jeu. Mieux, elle permet de tracer des orientations virtuelles indéterminées dans l'expérience. Dès lors, la pensée est plus attentive aux mouvements révolutionnaires en train de se faire, puisque la révolution n'est plus interprétée comme le terme de la réalisation progressive d'un mouvement réel, mais comme l'ensemble des lignes de fuite qui se dessinent dans l'ordre social et s'emploient à y tracer autant de lignes de fractures. Lignes de fractures dont on peut, mais seulement à titre réfléchissant, distinguer les forces respectives mais aussi les faiblesses, non pas à partir d'un principe transcendant (idéal d'un antagonisme radical), mais depuis une polarité tendancielle (plus ou moins de simplification) immanente aux mouvements et aux luttes de classes.

Il est vrai que, à l'instar du sens qu'il produit, cet usage réfléchissant est, en quelque sorte, un oiseau pour le chat : les virtualités qu'il soulève peuvent toujours être réinterprétées dans le cadre de la nécessité historique. Il est toujours possible, a posteriori,

Ibid., p. 120.

2

Ibid. 
de relever les écarts à la nécessité idéelle dans le mouvement dialectique de simplification. C'est d'ailleurs ce que fait Marx dans son quatrième et dernier article consacré à la conjoncture de 18481851 en France, repris comme chapitre IV aux Luttes de classes. Recensant les points de fracture discernés dans l'article précédent, Marx procède à une remise en ordre, à partir d'une nouvelle désillusion quant aux espérances révolutionnaires dont témoignait «Conséquences du 13 Juin 49 ». Au début du quatrième chapitre, Marx rend raison a posteriori, c'est-à-dire quelques mois après avril 1850 - octobre en l'occurrence - de l'échec de ces tendances révolutionnaires de mars 1850. Pour toute bonne économie matérielle dialectique, telle que celle depuis laquelle Marx écrit désormais, une fois l'indétermination refermée, l'effervescence révolutionnaire ne pouvait finir que de cette manière : par un échec. Et cela, notamment, en raison de la bêtise des Montagnards et des paysans, lesquels, bien qu'ils se soient rangés aux côtés du prolétariat, n'étaient in fine et en réalité pas assez mûrs : trop montagnards encore et donc petits-bourgeois réformistes prêts à s'engouffrer dans la première victoire parlementaire venue et à renoncer à l'usage insurrectionnel de la force ; trop paysans encore et donc incapables «d'initiative révolutionnaire ». ${ }^{1}$ À quoi il faut

Ibid., p. 132. Que dit exactement Marx dans ce chapitre ? En substance, que la situation économique en 1850 rendait impossible une véritable révolution, car cette situation était trop prospère, au sens où les forces productives se développaient sans pour autant mettre en question les conditions bourgeoises, sans entrer en conflit avec les formes de productions bourgeoises qui les conditionnent. Autrement dit, sur l'échelle de la simplification des antagonismes, mars 1850 était encore trop bas. Double conséquence : d'une part la lutte n'est pas radicalisée au point d'être rendue intolérable pour les dominés (ainsi, selon Marx, la raison majeure du non-soulèvement du prolétariat lors des mois qui suivent mars 1850 est due à la prospérité commerciale et industrielle), d'autre part, les classes coalisées du parti rouge n'ont pas été intégrées dans une classe opposée à la classe bourgeoise. La preuve en est que les classes rassemblées autour du prolétariat restaient bien des classes distinctes, autrement dit que la lutte restait trop plurielle encore, que la simplification n'avait pas amené les classes intermédiaires à se fondre dans la classe prolétarienne; dès lors, il ne fallait guère s'étonner si, dès la 
ajouter l'absence de radicalisation des conditions d'existence du prolétariat, c'est-à-dire le trop faible degré d'antagonisme. Le niveau de vie des prolétaires était relativement supportable ; il ne pouvait donc pas provoquer l'insurrection tant attendue. En somme, la contradiction n'était pas parvenue au point-limite révolutionnaire.

Nous sommes devant un mouvement rigoureusement inverse à celui qui entraînait le lecteur à la fin du chapitre III - soit : l'ironie plutôt que l'enthousiasme ? Pas de précipitation, il faut que la situation mûrisse. Ainsi, ce qui semblait permettre l'insurrection est aussi ce qui l'empêche d'être révolutionnaire. Pour Marx, toute précipitation est, en même temps qu'une dimension de l'explosion insurrectionnelle, l'indice d'une tentative toujours déjà vouée à l'échec. Objectivement, les conditions doivent être radicalisées pour que les intérêts des classes intermédiaires et du prolétariat soient identiques, à savoir unanimement orientés vers le renversement de la classe bourgeoise. Subjectivement, le prolétariat et le reste du peuple doivent effectuer leur apprentissage, passer par toutes les illusions et les désillusions, pour se rassembler enfin sous le bon mot d'ordre, celui de la vérité de l'avenir, et jouer à nu la pièce de l'aboutissement de la réalité historique. La célèbre rhétorique de la répétition dont Le Dix-huit Brumaire fera grand cas n'est rien d'autre que l'instrument par lequel le prolétariat et Marx lui-même éprouvent, c'est-à-dire mesurent, l'apprentissage des hommes à travers les conjonctures révolutionnaires ${ }^{1}$. Nous conclurons sur ce point.

moindre occasion, les divergences se sont fait jour : ainsi suite à sa victoire aux élections de mars, la Montagne a cru qu'elle pouvait désormais se contenter de cette voie légale - la Montagne n'a plus pris comme but l'émancipation du prolétariat, elle n'a plus pris non plus le parti de l'ordre comme un adversaire dangereux, comme un opposant qu'il fallait à tout pris renverser.

1 Sur la répétition historique chez Marx, en particulier dans Le Dix-huit Brumaire, on ne manquera pas de consulter l'incontournable et désormais classique ouvrage de P.-L. Assoun, Marx et la répétition historique, Paris, PUF, 1978. 


\section{Conclusion : répétition et apprentissage}

Partons d'un exemple a contrario : la Montagne. Plus précisément, la Montagne «puînée », comme l'appelle Marx, c'està-dire la Montagne de 1848-1852. Elle procède d'une double répétition. D'une part, elle répète la Montagne de 1793, c'est-à-dire, en quelque sorte, joue sur la scène révolutionnaire de 48 dans les costumes (attitudes, vocabulaires, etc.) de la Montagne de 1793, donnant cette impression - et se la donnant à soi-même - que la révolution de 1848 dans son ensemble répète - pour l'accomplir, pour la reprendre et l'achever - la Révolution Française, plus précisément la période de la Convention. D'autre part, la Montagne puînée ne cesse de se répéter elle-même, de rejouer les mêmes actions, voire de se plagier, de 1848 à 1852, reproduisant, en somme, les mêmes erreurs. Ce sont les deux aspects de la Montagne nouvelle comme répétition : volonté de reprendre le passé là où il avait été délaissé et d'en relancer la marche en le rejouant ; incapacité à tenir compte de ses erreurs et reproduction, quasi à l'identique, des postures, des actes et des discours qui la mènent tout droit dans des impasses à leur tour réitérées. Avec la Montagne puînée, tout se passe comme si le temps faisait du sur place, comme si le temps ne passait plus. Précisons néanmoins quelque peu les enjeux de l'analyse marxienne de cette double répétition.

La Montagne de 1848 veut reprendre la marche de l'histoire là où, à ses yeux, elle s'est arrêtée : la Montagne annule le temps luimême. Le problème, c'est que les acteurs de la période 1848-1852, eux, ont bel et bien éprouvé le passage du temps reléguant 1793 au passé. Les gestes de la Montagne apparaissent alors comme l'expression d'un désir stupide et improductif. D'où le ridicule, aux yeux des acteurs de la conjoncture - et, en premier lieu, aux yeux de Marx - de cette grandiloquence qui caractérise la Montagne, en particulier son représentant le plus fameux, Ledru-Rollin. Elle se révèle incapable d'être adéquate à son époque ; plus exactement, d'y être active et agissante. La question de la répétition permet ainsi à Marx de mettre en scène la capacité des acteurs à éprouver le passage 
du temps et à prendre acte de l'événement. Elle lui permet de tracer la ligne de démarcation entre ceux qui refusent la radicalité de l'événement et prétendent revenir en arrière ; et ceux qui s'efforcent de tenir l'événement et ses conséquences, c'est-à-dire la rupture du temps lui-même. Les premiers répètent ; les seconds avancent. Précisons néanmoins qu'il faudrait distinguer la manière dont la construction d'une durée propre à l'événement (c'est-à-dire son inscription sur une surface d'enregistrement qui est à construire) implique et passe par l'inscription simultanée de l'événement dans un code révolutionnaire qui lui est propre, d'un simple désir de retour dans le passé. La différence entre l'un et l'autre, c'est que la construction d'une durée propre de l'événement s'appuie sur un passé qui n'est pas tout entier déjà là et qu'il s'agit tout autant de construire. Par exemple, porter le slogan « République » est bien entendu pour les socialistes et les ouvriers une manière de reprendre un mot d'ordre déjà là sous la Restauration, et d'inscrire également leur lutte dans le cadre de 1789 ou 1793. Mais il ne s'agit pas pour autant de coder l'événement et de le réduire à un ordre temporel transhistorique qui s'est déjà manifesté, dans un passé qu'on peut rejoindre d'un bond. Reprendre un mot d'ordre, c'est une manière, par une référence explicite à 1793 et aux luttes de 1830 et d'après, d'ouvrir le temps et de le construire, c'est-à-dire moins d'annuler l'événement dans un ordre tout fait, "la révolution », que de le tenir par l'élaboration d'une mémoire des luttes.

La Montagne semble répéter certains faits au sein même de la conjoncture 48, comme si les premiers faits de l'insurrection ne cessaient de se répéter compulsivement. Pour comprendre cette seconde répétition, il faut la relier à la première. Ce sont les mêmes qui sont incapables d'agir leur temps et qui le répètent inlassablement ; ce sont les mêmes qui ne vivent leur temps que sur le mode d'un passé qui ne passe pas, qui n'est pas pour eux passé, où ils se réfugient, et qui ne parviennent pas plus à faire passer leur présent. Mais ce second aspect de la répétition apporte une idée nouvelle. Car il n'est pas sans paradoxe. Il revient, à force de répétition, à nier, d'un certain point de vue, l'idée même de 
répétition. De quel point de vue ? Du point de vue de celui qui répète. La condition de la répétition, c'est que celui qui l'effectue n'en soit pas conscient, parce qu'il n'est pas conscient des erreurs qu'il commet. La Montagne ne se rend pas compte que quelque chose a changé, parce qu'elle ne se rend pas compte qu'elle s'est trompée, qu'elle se bernait d'illusion en juin 1849. Les illusions de juin 1849 n'apparaissent pas à la Montagne comme illusions ; ses attitudes ne lui paraissent pas démenties par l'expérience, elles ne sont pas démodées à ses yeux ; subjectivement, rien n'a donc changé. On recommence donc juin 1849 en mars 1850. C'est pour les autres que la répétition existe, aux yeux desquels ce sont bien les mêmes gestes répétés dans deux situations différentes, dans deux moments du temps différents. Mais la Montagne vit dans un seul et même temps, celui qui lui permet de passer sans ambages d'une époque à une autre - de 1848 à 1793, de 1851 à 1848. Marx ne manquera pas de s'en moquer à travers des formules telles que : «la Montagne dupée une fois plus »? La Montagne n'a pas le sentiment du changement. C'est comme s'il ne s'était rien passé, c'est-à-dire très précisément : c'est comme si ses attitudes, actions, pensées, pratiques, etc., de juin 1849 n'avaient jamais existé, au sens où elles n'auraient jamais conduit à un échec. La question de la répétition permet à nouveau le tracé d'une ligne de démarcation : entre ceux qui ont appris et ceux qui n'apprennent pas, entre ceux qui mûrissent et ceux qui restent en enfance. Si la Montagne ne se rend pas compte de ses illusions, si elle ne voit pas qu'elle reproduit les mêmes erreurs qui l'ont conduite naguère à l'échec, si elle reste ignorante de ses propres répétitions, Marx, et le prolétariat selon Marx, quant à eux le voient. Or, si le prolétariat (et donc Marx) perçoit le changement, c'est précisément parce qu'il changé lui-même. Ce qui signifie que le concept de répétition fonctionne pour le prolétariat comme un moyen d'évaluer son propre changement. Alors que la Montagne répète les mêmes illusions, les prolétaires voient l'écart entre la situation nouvelle et les sempiternelles erreurs, tout comme ils perçoivent leur propre changement par opposition à l'immobilisme de la Montagne. Les répétitions sont pour le 
prolétariat différenciantes, c'est-à-dire productrices de différence d'une différence de temps, d'une transformation irréductible d'une situation en une autre, d'une rupture irréversible : d'un événement. Cette manière de vivre la répétition, Marx la pense dans Les luttes de classes en France dans les termes d'un apprentissage. Pour le prolétariat $^{1}$, la conjoncture de 1848-1851 fut une « école $»^{2}$.

La distinction des types ou des modes de répétition, en tant qu'elle permet de tracer une double ligne de démarcation, entre ceux qui tiennent et ceux qui refusent l'événement, et entre ceux qui apprennent et ceux qui répètent leurs erreurs ou plutôt stagnent dans leurs illusions, se révèle capitale. S'y joue en effet la sensibilité à l'historicité objective et subjective ; s'y joue la capacité à voir le changement et à changer soi-même. Si Les luttes de classes en France construisent plus qu'elles ne dévoilent une leçon des événements, c'est au sens où Marx y éprouve une rupture entre le passé et le présent en raison d'une épreuve plus profonde, celle de l'écart entre l'illusion et quelque chose comme une vérité - une vérité apprise. Il répète ainsi dans la théorie l'apprentissage pratique du prolétariat. Il l'accompagne dans l'apprentissage de la réalité de sa situation, c'est-à-dire du degré de simplification des antagonismes de classes qui structure le présent. Par là, Les luttes de classes en France fait bien l'apprentissage de la vérité. Mais cela reste l'apprentissage de la vérité de l'histoire en train d'advenir, avec ses

Et, en partie, pour la paysannerie. On relèvera sur ce point l'ambiguïté de Marx : d'abord élevée à l'école de la révolution comme le prolétariat (chapitre III), la paysannerie apparaît ensuite elle aussi incapable d'apprendre, prise dans une logique contre-révolutionnaire qui la précipite dans les bras du neveu Bonaparte, faisant figure de mauvais élève, responsable pour une bonne partie de l'échec du printemps 1850 et de la clôture progressive (ou plutôt, régressive) de la conjoncture révolutionnaire au profit de la réaction impériale. C'est d'ailleurs ce qui poussera Marx à esquisser une analyse plus fouillée des conditions socio-économiques et de la structure idéologique des "paysans parcellaires » dans le dernier chapitre du Dix-huit Brumaire. Voir K. Marx, Le Dix-huit Brumaire de Louis Bonaparte, op. cit., p. 257-268.

La formule revient sous la plume de Marx à plusieurs reprises dans Les luttes de classes en France. 
incertitudes et ses désillusions. Marx peut évaluer les positions de classe, le degré de contradiction, la possibilité de la révolution : la maturation de l'histoire. Il sait cependant que ses leçons ne sont pas définitives et qu'elles se compliquent à la rencontre des événements. Aussi lui faudra-t-il attendre et analyser la complexité du réel, dans toutes ses dimensions. Patiemment. Sans précipitation.

\section{Appendice : à propos de la configuration blanquiste. Remarques sur François Pardigon}

La lecture d'Auguste Blanqui et de François Pardigon nous a permis de tracer, moins une alternative à la position marxiste, qu'une autre tendance de la pensée des luttes et de leur temporalité, qu'il serait possible de greffer à celle de Marx, aux lieux de son discours où elle s'avère, en fait, plus compatible avec celui de ses esprits que nous avons choisi de privilégier, que ne le sont les énoncés marxistes eux-mêmes. Il nous semble utile d'esquisser les principaux motifs d'une ligne théorique et pratique peu connue et pourtant riche de sens et d'enjeux, en vue d'un travail ultérieur.

Reprenons les termes principaux structurant les possibilités d'interprétation d'un événement révolutionnaire. Le rétablissement de l'ordre et la négation de l'événement insurrectionnel se réalisent par son inscription sur une certaine surface. Mais pas seulement. C'est également la négation d'autres enregistrements possibles. Ainsi, l'inscription de 1848 par la bourgeoisie comme avènement de la République bourgeoise entraîne la négation de 1848 comme avènement de la République sociale, au moyen de la négation de la mémoire de la surface propre à la République sociale, en particulier de son référent majeur, 1793.

«Le Gouvernement provisoire, de son côté, une fois contraint de proclamer la République fit tout pour la rendre acceptable à la bourgeoisie et aux provinces. Les horreurs sanglantes de 
la $\mathrm{I}^{\mathrm{re}}$ République française furent désavouées par l'abolition de la peine de mort pour délits politiques $»{ }^{1}$

Pour le prolétariat, l'enjeu de la République, qui petit à petit devient l'organisation du travail, passe par l'enjeu de l'histoire dans laquelle elle s'inscrit. Si l'objectif de la bourgeoisie est bien d'« en finir avec les ouvriers $»^{2}$ et leurs revendications désormais officialisées, alors, selon Marx, il faut en finir avec l'histoire, avec les histoires dans lesquelles ils inscrivent leurs luttes et les revendications qui les portent, ainsi qu'avec les idéologies dans lesquelles elles baignent. Or cette question est la question même de François Pardigon.

Il nous semble en effet possible de trouver dans les réflexions de Pardigon les boutures pour une greffe appropriée - même si les éléments d'une position authentiquement insurrectionnelle dans la théorie (id est par rapport aux rémanences d'idéalisme) restent eux aussi pris et coagulés dans de nombreuses telles rémanences. Disonsle vite, d'emblée : les éléments d'une pensée non-dialectique de la conjonction des forces et non de leur progressif devenir-ce-qu'ellessont via le travail du négatif. Pensée de la rencontre et de l'exil.

Certes, chez Pardigon se retrouve exemplairement une compréhension profondément téléologique et idéaliste de la Révolution, faisant sourdement son chemin à travers l'histoire, avec et contre son refoulement par les obstacles absurdes de la contingence et les forces brutales de la Réaction. Mentionnant les révoltes consécutives au "guet-apens du 2 décembre », les insurrections populaires des petites villes, des bourgs et des hameaux formant comme un vaste «bataillon sacré » ${ }^{3}$, Pardigon les juge d'autant plus significatifs qu'ils sont au fond désespérés, " sans signal, sans chef, sans direction $»^{4}-$ à la fois éparpillés et voués à l'échec tant est importante, à ce moment, l'intensité de la répression

K. Marx, Les luttes de classes en France, op. cit., p. 24.

2 Ibid., p. 30.

3 Ibid., p. 97.

4 Ibid. p. 95. 
(état de siège pour un tiers des départements, propagande intensive, contrôle de tous les appareils idéologiques tournant à plein régime) ${ }^{1}$. Mais c'est justement par là-même qu'ils témoignent de quelque chose - parce qu'ils ne sont pas le produit d'une lutte unifiée et concertée, mais sans ordre, et parce qu'ils ne peuvent espérer aucun succès. Leur action témoigne du cheminement souterrain de l'Idée républicaine dans le Peuple.

«Depuis 1848, la république a dû pousser bien de nouvelles racines dans le pays pour qu'elle recrutât de si braves défenseurs, au milieu même de populations qui ne l'accueillirent, en février, qu'avec hostilité ou indifférence ». ${ }^{2}$

Sous l'éparpillement et l'irrationalité de ces mouvements capillaires, ce qui se signale c'est le développement progressif de l'Idée de république, qui est la cause secrète de ces insurrections « sans signal », l'unité secrète de ces insurrections «sans chef »sans tête, sans principe -, le telos secret de ces insurrections "sans direction ». De telles insurrections constituent, en outre, un événement chargé de sens, un signe ${ }^{3}$, précisément en cela qu'elles ne viennent pas du cœur de la révolution, les villes, et en premier lieu Paris, mais de ses marges, de gens dont l'enthousiasme insurrectionnel ne s'explique $\mathrm{pas}^{4}$, et même s'explique d'autant moins qu'ils étaient hostiles ou indifférents à la République au moment où il eût été pertinent de lui être favorable. Que leur rapport

Voir ibid., p. 94-97.

Ibid., p. 97.

$\mathrm{Au}$ sens strictement kantien du terme. Voir Emmanuel Kant, Le Conflit des facultés, dans Kant, Euvres philosophiques, III, éd. F. Alquié, p. 894 : dans ce texte célèbre, Kant appelle à la recherche d'un événement qui « ne doive pas lui-même être considéré comme cause de (...) progrès, mais seulement comme indication, comme signe historique (signum rememorativum, demonstrativum, prognostikon)».

Ce qui est essentiel dans la conception kantienne de l'événement, c'est que l'enthousiasme pour la révolution des gens qui n'y ont pas intérêt ne $s$ 'explique pas - c'est l'absence de causalité phénoménale de l'affect qui en conditionne la valeur de signe. 
affectif à la République se soit modifié dans un rapport inverse à l'évolution de la conjoncture qui, de favorable, devient défavorable, indique précisément que l'Idée de République a progressé selon son historicité propre dans le temps même où sa réalité historique - et donc les conditions concrètes de son instauration - se trouvait dans une phase régressive. D'où l'importance du récit et de la mémoire, non seulement des victoires, mais aussi et peut-être surtout des défaites, d'autant plus importantes qu'elles ne peuvent pas être relevées comme les moments d'une victoire à venir. Elles sont annonciatrices, comme signes, d'une progression immanente de l'Idée ${ }^{1}$.

De là que Pardigon puisse considérer ces révoltes paysannes de 1851 comme « le phénomène le plus significatif, le plus important pour l'avenir, de tous ceux qui sont apparus pendant cette première moitié du XIXe siècle $»^{2}$. Une telle affirmation n'est guère anodine à propos de « jacqueries » qui, du strict point de vue de la réalité politique, ont surtout servi au pouvoir bonapartiste à se légitimer et à légitimer son propre durcissement comme recours de la notabilité provinciale contre cet objet de toutes ses hantises ${ }^{3}$. Il faut bien comprendre, néanmoins : phénomène important parce que significatif, significatif pour l'avenir - de la politisation des masses rurales rejoignant pour la première fois le « grand courant des idées nouvelles et du mouvement révolutionnaire ${ }^{4}$. Un tel phénomène contribue aux yeux de Pardigon, et en quelque sorte in absentia, au devenir-prolétaire des masses rurales, ou plutôt à la fusion tendancielle des luttes de classe paysannes et citadines-prolétaires dans la lutte des classes, pour reprendre la distinction posée plus

Voir F. Pardigon, Épisodes des journées de juin 1848, op. cit., p. 107 : « Telle est l'histoire : le martyrologe des peuples ; un récit de catastrophes où s'abîment parfois des races entières pour léguer à leur descendance quoi ? une idée ».

2

Ibid., p. 98.

Voir la note d'A.Héricord, op. cit., p. 98, note 1.

Ibid., p. 98. 
haut. Nous disons in absentia, car de front commun il n'y en a pas, dans le réel. De devenir-lutte des classes de ces luttes de classes il n'y a pas, dans la Realpolitik du monde réel. Mais ce front et ce devenir, ils se font, eux-mêmes et leur propre historicité, dans cette caisse d'enregistrement et de mémorisation que sont les corps des dominés. Par le « riche tribut de persécutions » ${ }^{1}$ qu'ils ont payé, les paysans, les provinciaux ont eu leurs journées de Juin, retrouvant et rajeunissant «les traces sanglantes des transportés $»^{2}$. L'unité à venir des forces révolutionnaires se fait ici et maintenant, au présent, sous nos yeux, là même où elle n'a pas lieu, pas de lieu, dans le mélange du sang des morts passés, dans l'unité des morts qui se donnent la main et contractent un pacte. Au présent car Pardigon la voit, comme s'il y était, sous ses yeux :

« Nous ne voyons plus, dans la démocratie, qu'une forte phalange dont les soldats, tous égaux, tous frères, tous frappés, tous passés sous les mêmes fourches caudines, dévorent secrètement leur affront et attendent pour briser le joug l'heure marquée par les destins $»^{3}$

C'est en cela - en tant que « l'ouvrier des champs a rejoint l'ouvrier des villes »-que cet événement intrinsèquement provincial est, en fait, «un événement national $»^{4}$. Thèse d'une grande force théorique. Et hallucinatoire, si on la lit à la rigueur : c'est dans la mort et dans le deuil que l'ouvrier des champs a rejoint l'ouvrier des villes, et nulle part ailleurs - un événement national parfaitement invisible donc, le passage d'un seuil dans le devenir-révolutionnaire de la révolution qui s'opère - ni sur le terrain d'opération concret où des troupes en armes se rejoindraient, ni même par l'égalisation tendancielle des conditions socio-économiques d'existence - mais dans la mort et la mémoire, support d'inscription et caisse d'enregistrement d'une autre historicité.

Ibid.

2 Ibid., p. 99.

3 Ibid.

$4 \quad$ Ibid., p. 98. 
De ce point de vue, qui est celui de l'auto-éducation de la révolution que Marx pensait non à partir du deuil mais plutôt du phénomène de la répétition, il est intéressant de noter que Pardigon confère un rôle et une puissance toute particulière à l'exil. Pardigon insiste sur ce phénomène, principalement sur la déportation à NoukaHiva dans les Marquises, sur la transportation au camp de Lambessa en Algérie et au bagne à Cayenne - c'est-à-dire les trois principaux lieux de déportation pour les prisonniers politiques de la Seconde République puis du Second Empire -, mais aussi sur la simple proscription, abolie seulement en 1859, entraînant l'exil, que ce soit en Angleterre, aux États-Unis, ou dans d'autres pays. Pardigon en donne une description très poétisée : «pèlerinage perpétuel », « des groupes de proscrits sillonnent les chemins comme des pestiférés $»^{1}$, ils n'ont plus de lieu propre et sont condamnés au nomadisme, du Piémont en Suisse, d'Ostende à Douvres puis «sur les voies tracées par l'émigration irlandaise » les États-Unis². Mais, jetés dans la dispersion et l'errance, ils sont comme unis par ce qui les isole et les sépare, et en viennent à former une véritable Internationale de l'exil, effectivement et concrètement sans propriété, sans patrie, sans famille. Ils alimentent depuis le dehors les énergies vives d'une révolution dont le cœur s'est refroidi, mais dont « la force vitale persiste aux extrémités et alimente les centres exténués », écrit Pardigon d'une métaphore physiologique renversée ${ }^{3}$. En terre d'exil, ils se branchent sur toutes les énergies révolutionnaires qui avaient été éveillées dans le monde par la Révolution de Février, qui sont restées vivaces et peuvent la féconder à leur tour. Ils portent en eux, sur leur corps, à même leur condition d'exilé, le témoignage de la révolution; ils sont «le livre vivant de la République universelle » ${ }^{4}$.

Ibid., p. 99.

Ibid. p. 100.

Ibid., p. 101.

4 Ibid., p. 102. Pardigon écrit la page suivante : « Tous ces peuples écrivent leur page sur le livre de l'Humanité ; ils apportent tous un enseignement ; ils combattent tous, par un côté, l'ennemi commun et universel ; ils n'ont qu'à s'interroger pour se comprendre et pour décupler leur action », ibid., p. 103. 
Thèse paradoxale : la diaspora des républicains dans l'exil fabrique du commun, leur fabrique une unité qu'ils manquaient à se donner « chez eux », à la maison - l'exil est le «terrain commun » où la classe ouvrière se fait son auto-éducation, « des emprunts et une éducation réciproque $»^{1}$.

On a vu le rôle essentiel que jouait chez Marx le mouvement de simplification de l'antagonisme, c'est-à-dire de constitution réciproque et en face-à-face de la position révolutionnaire et de la position réactionnaire (on peut aussi bien dire : prolétariat vs bourgeoisie, travail vs capital...). Il est donc intéressant que Pardigon formule le même enjeu - l'auto-constitution de la classe universelle dans la définition corrélative de l'« ennemi universel »- mais en trouvant sa solution dans la positivité de l'exil et des conjonctions ou des rencontres propres à son espace vide : « rencontres multipliées de fugitifs, sur les grands chemins, sur les ponts des navires, sur toutes les frontières », " difficiles entretiens où chacun s'essaye à balbutier une langue rebelle », tout se passe à un niveau immédiatement international ${ }^{2}$. Il est clair qu'aux yeux de Pardigon, la révolution ne peut se constituer comme telle qu'à cette échelle d'abord internationale - c'est-à-dire en marge (en périphérie) de toute forme de souveraineté, de tout État-national que ce soit, à l'extérieur de tout espace constitué comme État, à même la frontière, dans les interstices de la politique internationale - pour refluer ensuite dans les espaces nationaux, et non pas, selon le cheminement inverse, par extension et diffusion d'une révolution qui s'opérerait au cœeur du capitalisme, là où la contradiction serait « mûre ». La chose ne peut être que suggérée ici, et il va de soi que le discours de Pardigon est sans doute plus celui d'un certain enthousiasme poétique que de la froide analyse des processus historiques - mais il est possible que toute la logique marxienne, jusque et y compris celle à l'intérieur de laquelle se trouve pris le Capital, reste fondamentalement enfermée à l'intérieur des limites (géographiques, conceptuelles, imaginaires) de

Ibid.

2 Ibid. 
l'État-nation et du schème de la souveraineté qu'il implique. Il est alors possible d'estimer toute la valeur de ces penseurs de la conspiration, du groupuscule plutôt que du parti, de la guérilla d'un petit nombre plutôt que de la guerre à front ouvert de deux camps déclarés, qui pourraient permettre de décloisonner, peut-être, la pensée marxienne de cet ancrage état-national et souverain : et la décloisonner, éventuellement, de l'intérieur.

Nous ne pouvons que l'esquisser ici. Trois points fondamentaux pourraient autoriser à trouver chez Pardigon, entre autres, un instrument pour pousser en avant l'un des esprits de Marx au détriment de certains autres. D'abord, une pensée de l'enregistrement historique des expériences révolutionnaires qui ne se fasse pas en référence à un temps idéal défini par la théorie et fixant, pour ainsi dire a priori, les degrés relatifs d'avancement et de retard des processus - tout en impliquant en dernière analyse logiquement la prévalence du lieu politique où la révolution doit se produire d'abord, et donc en arraisonnant la possibilité de la révolution à une certaine forme nationale - mais qui se construise en référence à la seule mémoire ouvrière elle-même, dans son corps et ses deuils, dans et par le sang versé ${ }^{2}$. Ensuite, une théorie de la constitution de l'antagonisme révolutionnaire par simplification du conflit qui ne soit pas soumise à une dialectique en dernière instance nécessitariste, mais qui se pense en termes pleinement positifs de conjonctions de forces, de rencontres et de contingences, et ce surtout à une échelle, ou plutôt dans un espace immédiatement international - depuis la marge et non depuis le cœur du système. Enfin, troisième élément à prendre en considération : l'auto-constitution d'une temporalité révolutionnaire. L'analyse (ou la fantasmatique) pardigonienne des événements se fait en référence à une exigence fondamentale : « Il est urgent que l'action révolutionnaire se produise d'une façon aussi unitaire, aussi intense que possible $»^{2}$. La différence d'inflexion avec

C'est la question de l'idéologie et de son rapport à la révolution qui est ici en jeu.

2

Ibid., p. 123. 
le marxisme se trouve peut-être là, qui commande l'ensemble des différences descriptives et prescriptives des lectures : à ce qu'il reste chez Marx d'appel à la patience du concept, à la nécessité d'éprouver le cheminement de l'esprit authentique de la révolution, dans son devenir-lui-même, à travers les phases douloureuses mais nécessaires de sa contradiction, avec tout le côté Bildungsroman que cela implique (à l'objet prêt, l'historiographie marxiste ne partage-telle pas finalement le même présupposé que les meilleurs tenants de «l'historiographie républicaine dominante $»^{1}$, tels M. Agulhon : toute cette histoire compliquée, tout ce bruit et cette fureur ne sont qu'un long et pénible apprentissage ?), s'oppose l'affect, absolument inverse, de l'urgence de l'action. Le trait caractéristique de la constellation blanquiste, en ce qu'il diffère du marxisme, serait alors le suivant : à ses yeux, la révolution est toujours déjà à l'ordre du jour, elle se confond avec le socialisme et le socialisme se confond avec elle. Plus même, c'est, pour Pardigon, cette tension permanente des forces dans l'effort de révolution qui est la condition à laquelle s'opèrent les synthèses précédemment mentionnées (constitution d'une unité de classe dans le deuil et la mémoire, constitution d'une identité de classe dans l'internationale de l'exil) :

"Que chacun, par l'énergie de la volonté, par la fixité, par la souveraineté du but, se dispose à peser sur les événements, de telle sorte qu'il reste des traces ineffaçables des choses accomplies, et non plus seulement des aspirations, des tâtonnements et du verbiage $»{ }^{2}$

Là encore, lisons en toute rigueur : ce qui décide du sens réel des actions révolutionnaires passées, ce n'est pas leur repérage théorique dans un système de coordonnées pré-établi, c'est l'action révolutionnaire présente, la puissance de la volonté qui s'y engage et qui, en fonction de son intensité maintenue, fait être son propre passé comme trace ineffaçable ou comme aspiration creuse. De l'action révolutionnaire on ne peut pas dire ce qu'elle a été, mais seulement

A. Héricort, « Une mémoire d'outre-tombe », ibid., p. 27.

2

Ibid., p. 123. 
ce qu'elle aura été en fonction de l'effort révolutionnaire qui, s'appuyant sur elle, la remet en jeu en permanence. Comme l'écrit Blanqui quelque part : «Laissons l'avenir à lui-même ${ }^{1}$. Ici aussi, Marx joue contre Marx, Marx qui se contente de laisser les morts enterrer les morts - quitte à leur prêter main forte à l'occasion pour ensevelir le " coup de main » avorté de 1848 - mais qui veut néanmoins, on s'en souvient, que « [1]a révolution sociale du XIXe siècle ne peut puiser sa poésie dans le temps passé, mais seulement dans l'avenir $»^{2}$.

Là se trouve, peut-être, le point d'opposition majeur de Blanqui à Marx, dans le cadre de la problématique que nous avons engagée, et qui tourne largement autour de la question de la temporalité révolutionnaire comme meilleur modèle que celui de la causalité. Pour Blanqui, la révolution est par définition à l'ordre du jour, ce qui tient peut-être à ce que Blanqui la pense en technicien plutôt qu'en théoricien. Sa question n'est jamais celle des conditions de possibilité de la révolution (ce qui, avec les massives inflexions nécessaires, reste la question, au moins selon l'une de ses tendances, l'un des ses « esprits » dirait Derrida, à partir de laquelle s'organise la problématique marxienne) ; sa question est : compte tenu du fait qu'il s'agit d'être toujours en train de faire la révolution, de pratiquer maintenant l'insurrection, comment s'y prendre ? Plus précisément, quoiqu'on fasse, il s'agit de le faire du point de vue de la révolution, d'être toujours dans un état et une situation révolutionnaires, étant entendu que la conjoncture modifie les manières de s'y prendre pour être en état de révolution. Donc : quelles sont les formes adéquates qu'impose la conjoncture à celui qui veut pratiquer au présent l'acte insurrectionnel ? De sorte que là même où le diagnostic est similaire, chez Marx et Blanqui, la position du problème à partir de laquelle le diagnostic est posée est fondamentalement différente : l'urgence de l'action contre la patience du concept.

Voir A. Blanqui, Maintenant, il faut des armes !, op. cit.

2 K. Marx, Le Dix-huit Brumaire de Louis Bonaparte, op. cit., p. 122. 